\title{
Millimeter Wave Imaging Architecture for On-The-Move Whole Body Imaging
}

\author{
Borja Gonzalez-Valdes, Member, IEEE, Yuri Álvarez, Senior Member, IEEE, \\ Yolanda Rodriguez-Vaqueiro, Student Member, IEEE, Ana Arboleya-Arboleya, \\ Antonio García-Pino, Senior Member, IEEE, Carey M. Rappaport, Fellow, IEEE, \\ Fernando Las-Heras, Senior Member, IEEE, and Jose A. Martinez-Lorenzo, Member, IEEE
}

Abstract-This paper presents a novel interrogation system that combines multiple millimeter wave transmitters and receivers to create real-time high-resolution radar images for personnel security screening. The main novelty of the presented system is that the images can be created as the person being screened continuously moves across a corridor where the transmitters and receivers, working in a fully coherent architecture, are distributed. As the person moves, the transmitters and receivers are sequentially activated to collect data from different angles to inspect the whole body. Multiple images, similar to video frames, are created and examined to look for possible anomalies such as concealed threats. Two-dimensional (2-D) and three-dimensional (3-D) setups have been simulated to show the feasibility of the proposed system. The simulation results in 2 -D have been validated using measurements.

Index Terms-Backpropagation imaging, checkpoint, fast Fourier transform (FFT), imaging systems, multistatic radar system.

\section{INTRODUCTION}

$\mathbf{I}$ $\mathrm{N}$ homeland security applications, there is an increasing demand for methods to improve personnel screening for concealed object and contraband detection at security checkpoints. In this context, active nearfield millimeter-wave (mm-wave) imaging radar systems are able to provide highresolution imaging at an affordable cost. The object of interest is first illuminated by mm waves and then the scattered field is

Manuscript received June 18, 2015; revised January 11, 2016; accepted February 18, 2016. Date of publication XXXX XX, XXXX; date of current version XXXX XX, XXXX. This work was supported in part by the Ministerio de Ciencia e Innovacin of Spain/FEDER under project MIRIIEM-TEC201454005-P, in part by the Gobierno del Principado de Asturias through the PCTI 2013-2017, GRUPIN14-114, in part by the Spanish Government under project TACTICA, in part by the European Regional Development Fund (ERDF), in part by the Galician Regional Government under Projects CN2012/279, CN2012/260 (AtlantTIC) and the Plan I2C (2011-2015), and in part by the Science and Technology Directorate, U.S. Department of Homeland Security under the Award Number 2008-ST-061-ED0001.

B. Gonzalez-Valdes, Y. Rodriguez-Vaqueiro, and A. García-Pino are with the Atlantic Research Center, Universidad de Vigo, 36310 Vigo, Spain (e-mail: bgvaldes@com.uvigo.es; yrvaqueiro@com.uvigo.es; agpino@com.uvigo.es).

Y. Álvarez, A. Arboleya Arboleya, and F. Las-Heras are with the Area of Signal Theory and Communications, Department of Electrical Engineering, Universidad de Oviedo, E-33203 Gijón, Spain (e-mail: yalopez@tsc.uniovi.es; aarboleya@tsc.uniovi.es; flasheras@tsc.uniovi.es).

C. M. Rappaport and J. A. Martinez are with the ALERT Center, Northeastern University, Boston, MA 02115 USA (e-mail: rappapor@ece.neu.edu; jmartine@ece.neu.edu).

Color versions of one or more of the figures in this paper are available online at http://ieeexplore.iee.org.

Digital Object Identifier 10.1109/TAP.2016.2539372 measured and processed to reconstruct the surface (or volume) of the object.

The development of checkpoints that allow high passenger flow is becoming a priority. This has motivated the design of mm-wave imaging systems that minimize passenger inconvenience.

The International Air Transport Association (IATA) has defined several specifications that future checkpoints for personnel screening should meet. Novel paradigms in the design of the checkpoints specify that "from 2020 and beyond it is envisaged that the passenger will be able to flow through the security checkpoint without interruption unless the advanced technology identifies a potential threat," [1] (page 14).

In [1], a computer graphics design of the checkpoint of the future proposed by IATA is presented. The novelty with respect to existing architectures is the inclusion of a beltway or hallway to avoid passenger flow interruption.

Current state-of-the-art mm-wave imaging systems for security screening require people to enter and stand in front of the scanning system. Mm-wave generation and acquisition can be achieved using static arrays of transmitters and receivers [2], [3], or using movable arrays to create planar [4], [5], or cylindrical [6]-[8] acquisition domains. Most of them are based on monostatic radar and Fourier inversion [2]-[6]. Monostatic imaging systems are cost effective, but they are only able to reconstruct surfaces that create specular reflection and they are not well suited for imaging scattering objects with sudden profile variations [9]. Further, they are prone to dihedral artifacts as described in [8], [10], and [11].

Based on the new checkpoint architecture proposed by the IATA, this paper introduces a novel concept for mm-wave scanning system for personnel screening. The proposed imaging system does not include any mechanical movement, and whole body imaging is obtained taking advantage of the movement of the person under test when passing through the system on a moving walkway.

The main contribution of this paper is the introduction of this novel architecture, called on-the-move imaging [12], [13], that, to the best of the author's knowledge, has not been previously conceived nor demonstrated.

This paper is structured as follows. Section II describes the proposed mm-wave screening system. Imaging algorithm for multistatic setups is briefly described in Section III. Proof-ofconcept is validated through two-dimensional (2-D) simulation 

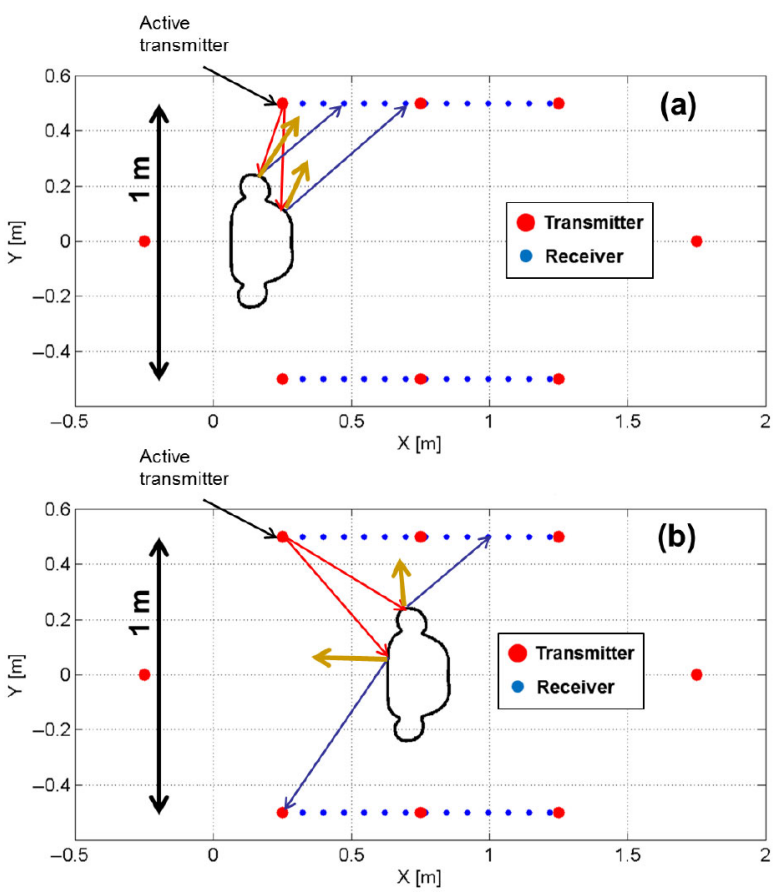

F1:1 Fig. 1. On-the-move imaging concept. OUT movement between the two walls F1:2 of radar antennas provides multiple points-of-view for every transmitter and F1:3 receiver, thus increasing multistatic information. (a) and (b) represent two F1:4 different OUT positions within the hallway. examples in Section IV, and measurement results in Section V. Extension to two-dimensional (3-D) whole human body imaging is described and validated in Section VI. Finally, the conclusion is presented in Section VII.

\section{ON-The-Move Hallway CONCEPT}

The novel mm-wave on-the-move imaging system for personnel screening takes advantage of: 1) the movement of the person when passing through the imaging system and 2) a mulstitatic radar configuration, where some of the transmitters and receivers are separated with a subtended angle relative to the person equal or greater than $90^{\circ}$ to capture information from all possible wave incident and scattering angles.

A top view of the suggested multistatic architecture is plotted in Fig. 1. Several transmitters (red dots) and receivers (blue dots) are placed on the sides of the hallway. The person moves along the security checkpoint on a moving walkway.

The imaging radar system takes advantage of multiple incidence angles that illuminate different areas of the person depending on the active transmitter and the placement of the person within the hallway, as illustrated in Fig. 1. A single transmitter can illuminate different areas of the person while crossing the hallway. Reciprocally, the scattered field is collected by different receivers depending on the transmitting element and the current position of the person. This is illustrated with the red and blue arrows in Fig. 1 that represent direct reflection contributions given by the incident angle and the normal to the surface according to Snell's law.

Multistatic information can be incremented by placing transmitters at the hallway ends. For practical implementation, this
TABLE I

COMPARISON With StATE-OF-THE-ART MM-WAVE IMAGING Systems

\begin{tabular}{|c|c|c|c|c|}
\hline Reference & $\begin{array}{l}\text { Scanning } \\
\text { area } \\
(\mathrm{cm})^{1}\end{array}$ & $\begin{array}{l}\text { PSF } \\
(\mathrm{mm})^{2}\end{array}$ & $\begin{array}{l}\text { Frequency } \\
\text { band } \\
(\mathrm{GHz})\end{array}$ & $\begin{array}{l}\text { Number of } \\
\text { antennas }\end{array}$ \\
\hline $\begin{array}{l}\text { On-the- } \\
\text { move }\end{array}$ & $100 \times 200^{3}$ & $10 \times 10$ & $15-30$ & $\begin{array}{l}2 \times 80601 \mathrm{Rx} \\
60 \mathrm{Tx}\end{array}$ \\
\hline $\begin{array}{l}\text { UWB MIMO } \\
\text { array, [5] }\end{array}$ & $50 \times 130$ & $10 \times 10$ & $2.8-19.5$ & $\begin{array}{l}4 \mathrm{Tx} \quad 8 \mathrm{Rx}, \\
\text { Height mo- } \\
\text { tion. }\end{array}$ \\
\hline $\begin{array}{l}\text { Flat 2-D array, } \\
\text { [2] }\end{array}$ & $100 \times 200$ & $3.0 \times 1.5$ & $72-80$ & $\begin{array}{ll}3072 & \mathrm{Tx} \\
3072 \mathrm{Rx} & \end{array}$ \\
\hline $\begin{array}{l}\text { Linear array, } \\
\text { vertical } \\
\text { movement } \\
{[4]}\end{array}$ & $\begin{array}{l}72.6 \\
\text { Movable } \\
2 \mathrm{~m} \text { in } \\
\text { height }\end{array}$ & $10.0 \times 3.8$ & $27-33$ & $\begin{array}{l}66 \mathrm{Tx}, \quad 66 \\
\mathrm{Rx}, \mathrm{Height} \\
\text { motion }\end{array}$ \\
\hline
\end{tabular}

${ }^{1}$ Scanning area size: width $\times$ height.

${ }^{2}$ PSF (point spread function): range $\times$ cross range.

${ }^{3}$ Receiving panels size.

would partially block the persons path. This is solved in the 3-D 105 case placing the receivers at the hallway ends below and above 106 the moving walkway.

For every transmitter, the scattered field is collected on the 108 receiving arrays placed on the hallway sides, and for every 109 receiving array, a reflectivity image is recovered. The reflectiv- 110 ity images associated with each transmitter are coherently com- 111 bined. This configuration assumes that, for a single position, 112 the body remains still while all the transmitters are sequentially 113 activated and the scattered field is collected by the receivers. 114 In this sense, and since the acquisition on the receivers can be 115 done in parallel, the use of a low number of transmitters is desir- 116 able. A fully electronic scanning system similar to the one in [3] 117 would easily allow for such an acquisition procedure.

A critical aspect in the design of the imaging system is the 119 selection of the frequency band. Table I shows a comparison 120 among the proposed hallway concept and some of the exist- 121 ing mm-wave scanning systems. It can be observed that, for 122 a given size of the scanner, higher frequency bands provide 123 better cross-range resolution, at the expense of losing dynamic 124 range due to free-space propagation losses. Furthermore, cloth- 125 ing becomes less transparent for these higher frequency bands, 126 and radiofrequency hardware becomes more expensive. The 127 work presented in [5] addresses the aforementioned drawbacks 128 introducing an ultra-wideband (UWB) imaging system. In addi- 129 tion to the improved range resolution and dynamic range, the 130 novelty of this study is the fact that the sampling rate can be 131 relaxed by taking advantage of grating lobes cancellation in 132 UWB arrays, which will be of interest concerning practical 133 implementation of the on-the-move architecture.

\section{IMAGING ALGORITHM}

Practical mm-wave scanning system implementation 136 demands real-time imaging capabilities. Standard backprop- 137 agation techniques [14] require millions of calculations for 138 electrically large acquisition and imaging domains. To illus- 139 trate the numerical magnitude of the problem, typical values 140 for acquisition points and imaging voxels are $10^{5}$ and $10^{7}, 141$ 
respectively, assuming an operational frequency of $30 \mathrm{GHz}$ $(\lambda=1 \mathrm{~cm})$ and sampling every half wavelength in both domains according to Nyquist criterion.

The reflectivity function on a volumetric domain $\rho_{t}\left(x^{\prime}, y^{\prime}, z^{\prime}\right)$ can be recovered from the scattered field $E_{\text {scatt }}^{t}(f, x, z)$ acquired on a flat receiving aperture placed at $y=Y_{0}$, by solving the following integral equation [9], [14], when the $t$ th (with $t$ from 1 to $N_{t x}$ ) of a group of transmitters is active

$$
\begin{aligned}
& \rho_{t}\left(x^{\prime}, y^{\prime}, z^{\prime}\right) \\
&= \iiint E_{\text {scatt }}^{t}(f, x, z) e^{+j k\left(\left(x-x^{\prime}\right)^{2}+\left(Y_{0}-y^{\prime}\right)^{2}+\left(z-z^{\prime}\right)^{2}\right)^{1 / 2}} \\
& e^{+j k\left(\left(x_{i n c}^{t}-x^{\prime}\right)^{2}+\left(y_{i n c}^{t}-y^{\prime}\right)^{2}+\left(z_{\text {inc }}^{t}-z^{\prime}\right)^{2}\right)^{1 / 2}} d f d x d z
\end{aligned}
$$

where $\left(x_{i n c}^{t}, y_{i n c}^{t}, z_{\text {inc }}^{t}\right)$ denotes the position of the $t$ th point source-like transmitter, $k=2 \pi f / c$, y-axis is the range axis (depth), $\mathrm{x}$ - and $\mathrm{z}$-axes are horizontal and vertical cross ranges, and $f$ is the frequency.

Fast propagation techniques, such as the inverse fast multipole method, have been proposed [15], reducing the calculation time by several orders of magnitude. Moreover, (1) can be parallelized taking advantage of GPU hardware. However, these solutions are still too computationally expensive for applications requiring real-time imaging.

Fourier-based techniques have been widely used in monostatic setups for real-time imaging [3]-[5], thanks to the fact that plane wave incidence can be considered during the inversion. Multistatic setups require different Fourier processing as the transmitter and receiver are placed in different positions. A novel Fourier-based imaging technique, totally suitable for the proposed hallway-based on-the-move imaging system, is presented in [9]. The idea is to decompose the imaging domain in smaller regions where an incident spherical wave can be locally treated as a plane wave. Imaging calculations for every region can be carried out in parallel, without jeopardizing the required real-time capabilities of the multistatic imaging system.

When multiple transmitters are used, the final reconstruction for a certain voxel placed in $\left(x^{\prime}, y^{\prime}, z^{\prime}\right)$ can be obtained by combining the images generated by each transmitter as

$$
\rho\left(x^{\prime}, y^{\prime}, z^{\prime}\right)=\sum_{t=1}^{N_{t x}} \rho_{t}\left(x^{\prime}, y^{\prime}, z^{\prime}\right) \text {. }
$$

This formulation assumes all the transmitters and receivers work in a fully coherent configuration using a clock signal that provides common phase reference.

\section{2-D RESULTS}

The proposed on-the-move imaging is first validated using a 2-D example. The frequency band ranges from 15 to $30 \mathrm{GHz}$, sampled every $300-\mathrm{MHz}$ frequency steps and providing $1-\mathrm{cm}$ range resolution. Two 1-m width lateral arrays of receivers with 50 evenly spaced elements are placed at $Y_{0}=-0.6 \mathrm{~m}$ and $Y_{0}=0.6 \mathrm{~m}$. Five transmitters are interleaved among each

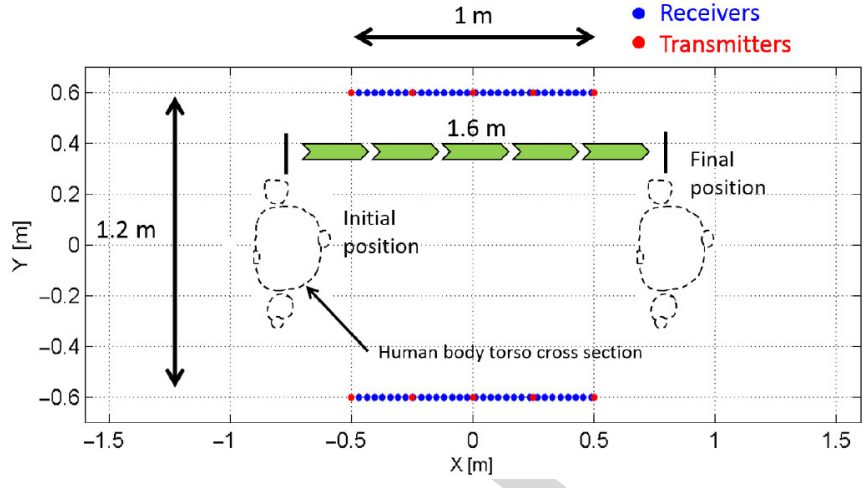

Fig. 2. 2-D example layout. OUT is displaced from position $x=-0.8 \mathrm{~m}$ to $\mathrm{F} 2: 1$ $x=+0.8 \mathrm{~m}$, in five steps $\left(N_{\text {pos }}=5\right) .5$ Tx and 50 Rx per side are considered. F2:2

panel of receivers, thus resulting in $N_{t x}=10$ transmitters. The 186 described layout is plotted in Fig. 2. The essential aspect is that, 187 in order to image the entire body surface, for every transmitter, 188 receivers on both walls must receive the scattered waves (not 189 just those adjacent to a given transmitter.)

The object under test (OUT) models the cross section of 191 a human body torso (for a more realistic simulation, arms, 192 and waist are not connected), with three attached objects on 193 it represented as protrusions on the front, back, and arm. The 194 object in the front is an elliptical cross-sectional metallic object. 195 Dielectric objects $\left(\epsilon_{r}=3.5\right)$ are placed on the back (square 196 cross section) and on the right arm. The OUT is displaced 197 from the position $x=-0.8 \mathrm{~m}$ to $x=0.8 \mathrm{~m}$ in $40-\mathrm{cm}$ steps 198 obtaining $N_{\text {pos }}=5$ intermediate positions. For every position, 199 the ten transmitters are sequentially activated and the scattered 200 field is collected in the receiving points. A realistic compo- 201 sition of the human body tissue is considered [16], using a 202 finite-difference frequency-domain (FDFD) code [17], [18] to 203 calculate the scattered field for every transmitter and every OUT 204 position. FDFD simulation results have confirmed that, due to 205 the high conductivity of the skin in the frequency band of inter- 206 est, the assumption that the OUT is a perfect electric conductor 207 (PEC) is a good approximation for most cases. 208

The data are then used to create one reflectivity image for 209 each intermediate position, $\rho^{p}$ according to (1) and (2). The 210 imaging domain is an $(X, Y)=(0.4,0.6) \mathrm{m}$ rectangle, dis- 211 cretized in $81 \times 121$ pixels and centered in $\left(x_{p}^{\prime}, y_{p}^{\prime}, z_{p}^{\prime}\right)$. In this 212 case, the computational cost is low and the image is recovered 213 using the standard backprogation algorithm in (1). For every $p$ th 214 OUT position and $t$ th active transmitter, the image is recovered 215 in about $1 \mathrm{~s}$ using a conventional laptop (2.5-GHZ CPU and 4- 216 GB RAM memory). As the 2-D imaging code is not parallelized 217 yet, it takes about $50 \mathrm{~s}$ for the entire reconstruction. 218

The obtained images for two different active transmitters 219 when the OUT is in each of the intermediate positions are pre- 220 sented in Fig. 3. It is clear that each transmitter allows the 221 reconstruction of different areas of the body depending on its 222 relative position inside the imaging system. The image obtained 223 for the central position, combining the images created using all 224 the transmitters according to (2), is presented in Fig. 4. 

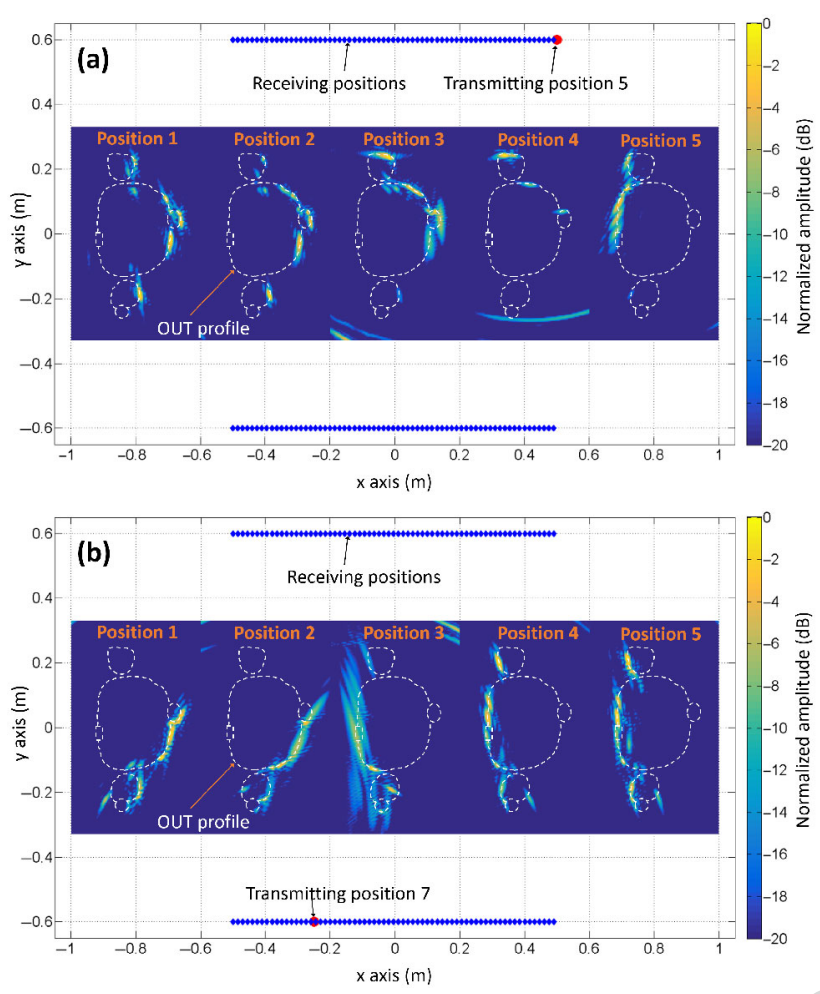

F3:1 Fig. 3. Obtained images (normalized reflectivity amplitude in $\mathrm{dB}$ ) for two difQ1 F3:2 ferent active transmitters and five intermediate positions using the setup in F3:3 Fig. 2. Active transmitters are depicted as red points. Blue points represent F3:4 receivers positions.

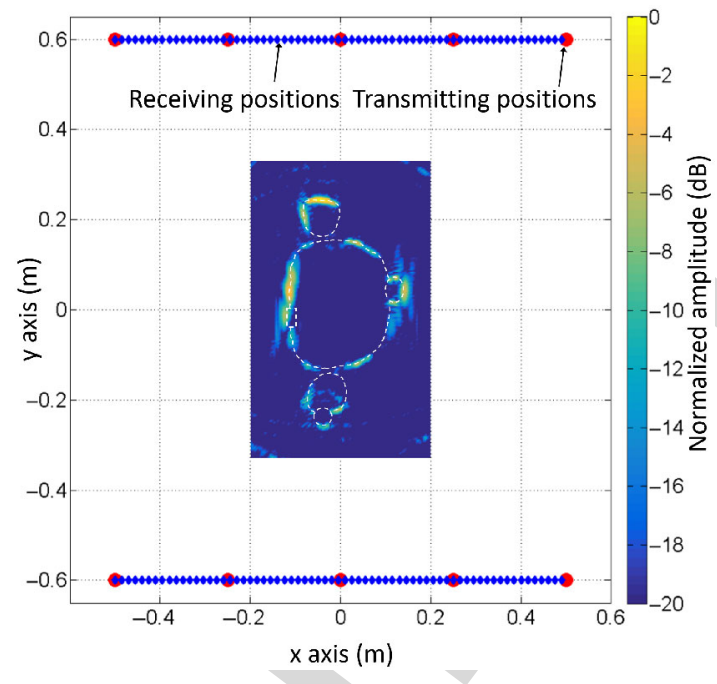

F4:1 Fig. 4. Obtained image when the OUT is in the central position and the image F4:2 is created using all transmitters according to (2).
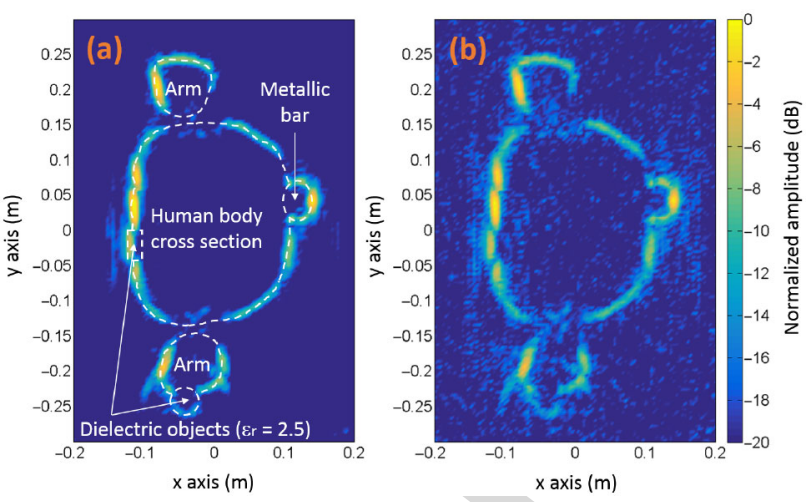

Fig. 5. Recovered OUT profile when combining in amplitude the five images F5:1 (one for each position). (a) $\mathrm{SNR}=10 \mathrm{~dB}$. (b) $\mathrm{SNR}=-20 \mathrm{~dB}$.

combination of the images of each position in amplitude and 232 phase. Fig. 5 presents the final result when the five analyzed 233 positions are combined according to (3), and when the object 234 retains exactly the same configuration for all positions and it is 235 only displaced in the $\mathrm{x}$ direction. This proves the ability of the 236 proposed system to obtain a complete contour reconstruction. 237 In general, the images used for threat detection in a final con- 238 figuration would be the ones generated in each position as the 239 one in Fig. 4.

Combining the information from multiple transmitters and 241 positions also helps to increase the dynamic range of the sys- 242 tem. Sensitivity analysis has been performed: first, the recorded 243 signal strength in the receiving arrays for every transmitting ele- 244 ment and OUT position has been evaluated. The case in which 245 maximum power is recorded corresponds to the OUT at the 246 central position illuminated by the center transmitters. The min- 247 imum power levels are recorded for the OUT in positions 1 or 248 5 illuminated by the closest pair of transmitters, as only a small 249 fraction of the scattered field is collected by the arrays. The 250 received power difference between these two cases is $11 \mathrm{~dB}$. 251

Next, noise has been added to the field samples accord- 252 ing to different signal-to-noise ratio (SNR) levels relative to 253 the maximum-recorded power case. Figs. 3-5(a) correspond to 254 $\mathrm{SNR}=10 \mathrm{~dB}$, and Fig. 5(b) to SNR $=-20 \mathrm{~dB}$. Thanks to the 255 combination of multiple OUT positions and incident directions, 256 the resulting mm-wave imaging system is able to work with low 257 SNR.

The capability of imaging multiple users within the hallway 259 has been also evaluated. For this purpose, the OUT placed at the 260 center position (as in Fig. 4) is considered, but with two more 261 OUTs (with no attached objects) at $x=0.7$ and $x-0.7 \mathrm{~m}$, a 262 scenario that could correspond to a high passenger through- 263 put situation. Due to the use of FDFD simulations, multiple 264 reflections among OUTs are considered. Results are depicted 265 in Fig. 6. It can be noticed that, with respect to Fig. 4, the cen- 266 ter OUT is worse imaged due to the multipath effects. It it also 267 possible to create the image of the front and the back of the 268 OUTs placed at $x=0.7$ and $x=-0.7 \mathrm{~m}$, and these results are 269 not affected by multipath as much as the center OUT.

In order to compare this work with current state of the art 271 systems, Fig. 7 presents the obtained image when the same con- 272 tour is facing a line containing the transmitters and receivers. In 273 


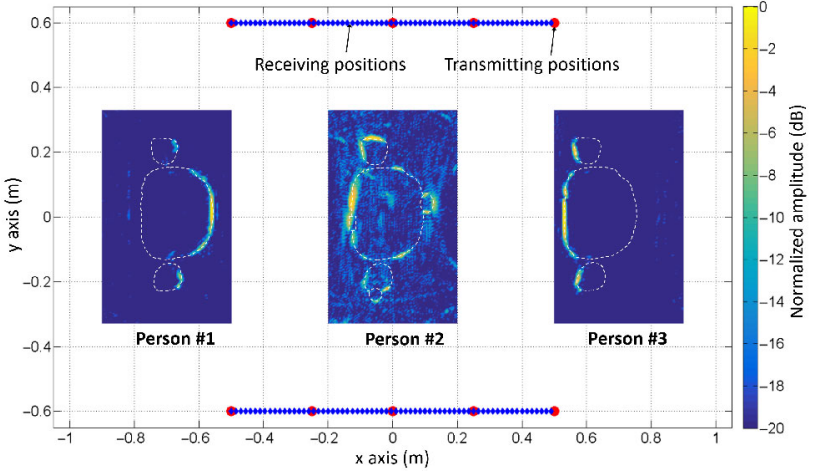

F6:1 Fig. 6. Recovered image for three OUTs placed at the same time in the hallway.

F6:2 The image is created by combining all transmitters according to (2).

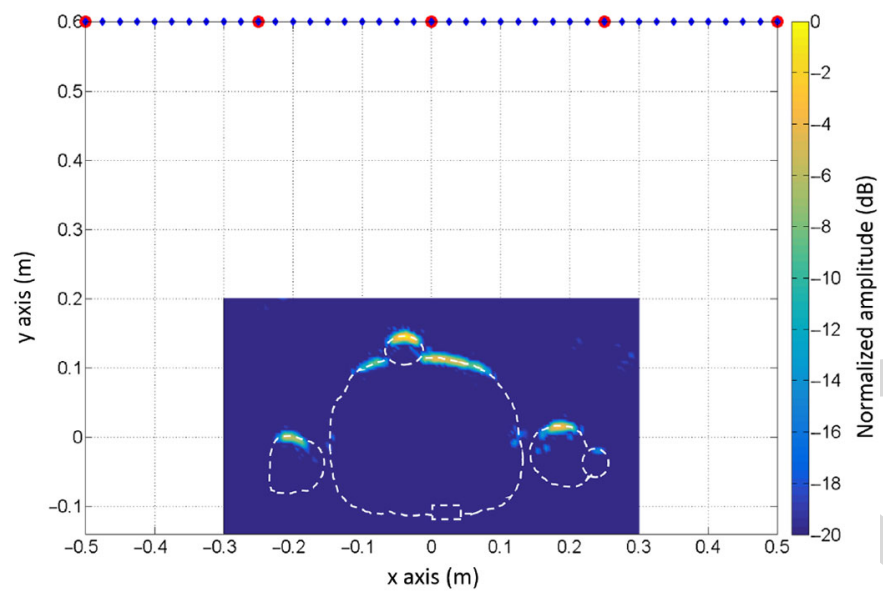

F7:1 Fig. 7. Obtained image using state-of-the-art configurations where transmitters F7:2 and receivers are placed in the same aperture and facing the person under test. F7:3 The image is generated combining the five transmitters according (2). this case, different areas of the front of the contour cannot be recovered and the area that is reconstructed is much smaller than the one of Fig. 4. Concerning detection capabilities, note that the dielectric object placed on the arm is hardly detected in Fig. 7 as the energy is not scattered back to the receiving array. In the case of the on-the-move system, it can be better detected (see Figs. 4 and 5), as it is possible to find a configuration along the conveyor belt in which the energy is reflected in the dielectric-skin transition, then backscattered to one of the receiving arrays.

This 2-D example proves that, in the proposed on-the-move layout, the fact that some of the transmitters and receivers are separated with a subtended angle relative to the person equal or greater than $90^{\circ}$ provides information from all possible wave incident angles.

\section{Validation With Measurements}

The proposed on-the-move imaging concept has been validated with measurements. Ka frequency band (26.5-40 GHz) has been selected to avoid hardware switching between different frequency bands. In order to ensure the maximum illumination within the hallway, WR-28 open-ended waveguides are selected as antennas.

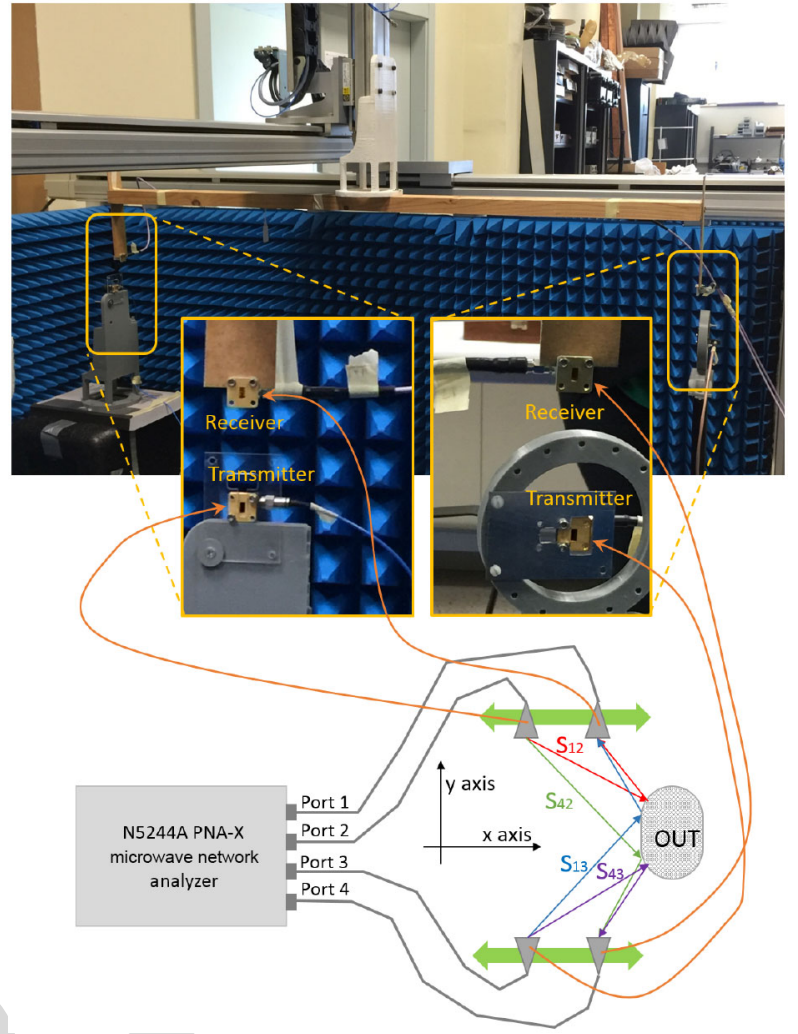

Fig. 8. Ka-band measurement system for on-the-move concept experimental F8:1 validation. WR-28 open-ended waveguides are connected to the vector network F8:2 analyzer ports. Receivers are mounted on a three-axis positioner.

F8:3

The setup is mounted on an XYZ table measurement range 296 [19], so some mechanical restrictions apply to the placement 297 of the OUT, transmitters, and receiving positions (Fig. 8). In 298 order to take advantage of the whole span of the XYZ measure- 299 ment range, scattered field samples are collected in 161 points 300 ranging from $x=-0.6 \mathrm{~m}$ to $x=0.6 \mathrm{~m}$, placed at $Y_{0}=0 \mathrm{~m} 301$ and $Y_{0}=1.3 \mathrm{~m}$. Five transmitting positions are interleaved 302 among the receivers, thus resulting in $N_{t x}=10$ transmitting 303 positions. Transmitters and receivers are separated $5 \mathrm{~cm}$ in 304 height. Horizontal polarization is considered to reduce coupling 305 between transmitter and receiver. The imaging setup is depicted 306 in Fig. 8: two transmitters and two receivers are connected to 307 the ports of a vector network analyzer. The power reference 308 level is $0 \mathrm{dBm}$ for all the ports. For every receiving position 309 along the $\mathrm{x}$-axis, four S-parameters are measured, as shown in 310 Fig. 8, corresponding to the combination of each transmitter 311 $\begin{array}{ll}\text { with both receivers. } & 312\end{array}$

The positioner of the XYZ table is used to move the receivers 313 from each side of the hallway at the same time, as shown in 314 Fig. 8. The pair of transmitters is manually placed at five posi- 315 tions along the $\mathrm{x}$-axis, using the $\mathrm{XYZ}$ positioner as reference. 316 For every pair of transmitting positions, acquisition time takes 317 $3 \mathrm{~min}$, and therefore, overall acquisition time for every OUT 318 position is $15 \mathrm{~min}$.

The OUT, shown in Fig. 9, is an aluminum foil-covered plas- 320 tic bin with a metallic bar attached to one of the sides. Due 321 to its translation symmetry in z-axis, it allows for 2-D analysis 322 in an XY plane placed at $\left(z=h_{t x}+h_{r x} / 2\right)$, where $h_{t x}$ is the 323 


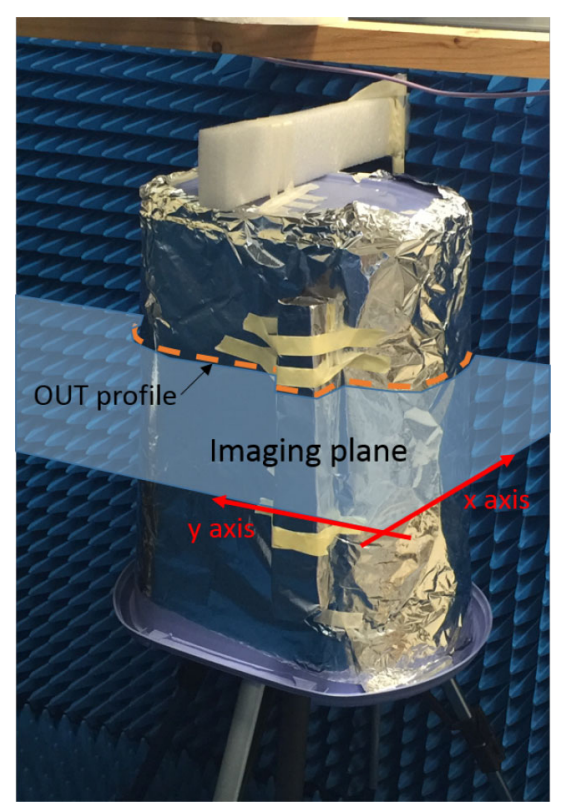

F9:1 Fig. 9. Photograph of the OUT imaged with the proposed experimental setup. F9:2 Receivers are mounted on a three-axis positioner.

height of the transmitters, and $h_{r x}$ the height of the receivers. As mentioned in Section II, using metal to simulate the human body skin in the Ka band is an acceptable approach due to the high conductivity of the skin in mm-wave frequency bands [16]. Three positions of the OUT were considered.

The same data processing as in Section III has been applied. The image obtained for every position, combining the images created using all the transmitters according to (2), is depicted in Fig. 10(a). It can be noticed that, for positions 1 and 3, the front and the back of the OUT are imaged, and the sides of the OUT are visible for position 2 .

Fig. 10(b) presents the final result combining the three OUT positions according to (3), where the OUT profile can be observed. In this case, combination is done taking the displacement of each individual image with respect to the center of the imaging domain. In practical, combination of the radar images for different positions of the person in the hallway can be based on video frames, linking video, and radar images.

In addition to the presented results, the measurement setup has been simulated, aiming to evaluate the correspondence between simulations and measurements. Results for position 2 are compared in Fig. 11. Good agreement between the reconstructed parts of the OUT for simulations and measurements is obtained.

\section{3-D CONFIGURATION}

Next, the extension from 2-D to 3-D is presented. The layout of the proposed on-the-move 3-D system is presented in Fig. 12. The setup is composed of multiple synchronized transmitters and receivers. Lateral receiving apertures of size $(\mathrm{X}, \mathrm{Z})=$ $(1,2) \mathrm{m}$, are placed at $Y_{0}=0.75 \mathrm{~m}$. The size of the panels is chosen to provide an approximated cross-range resolution of $1 \mathrm{~cm}$ along the $\mathrm{z}$-axis and $2 \mathrm{~cm}$ in the $\mathrm{x}$-axis.

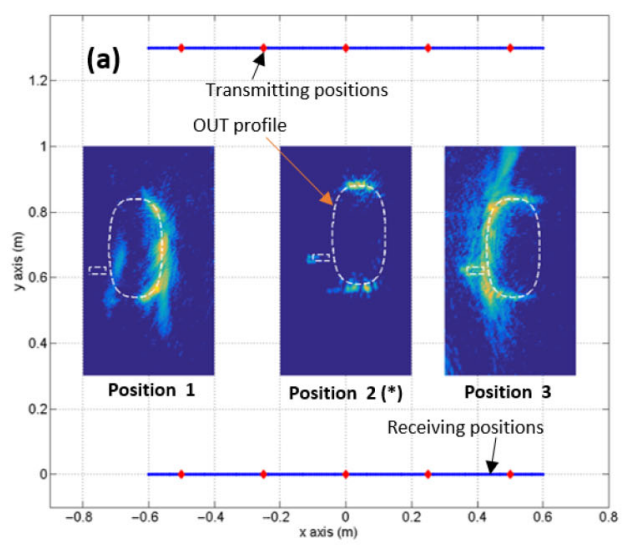

(b)

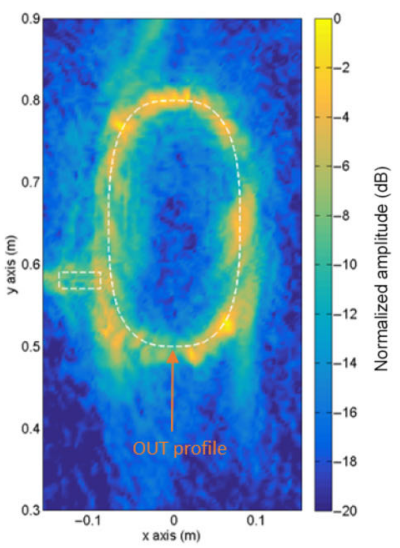

Fig. 10. Recovered OUT profile. (a) Image created on every position using F10:1 all the transmitters according to (2). In the case of position 2, only the cen- F10:2 ter transmitting positions $\left(x_{i n c}^{t}=0 \mathrm{~m}\right.$ ) were available. (b) OUT profile when F10:3 combining in amplitude the three images of (a).

$\mathrm{F} 10: 4$
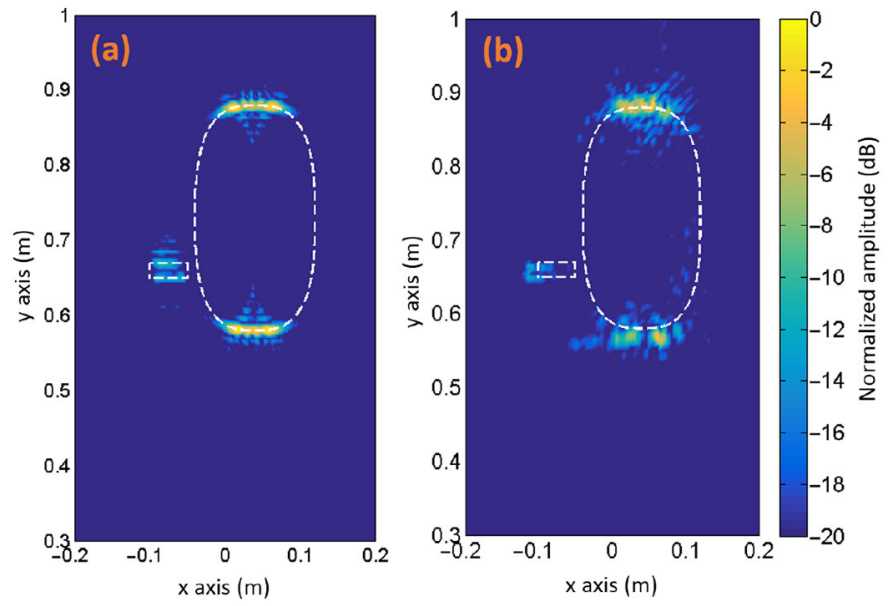

Fig. 11. Recovered OUT profile, position 2 (with center transmitting posi- F11:1 tions). (a) From simulated data. (b) From measurements.

F11:2

For this preliminary setup, Nyquist sampling requirements 356 are considered for the receiving panels, thus acquiring the field 357 in $201 \times 401$ receiving positions per panel. Subsampling tech- 358 niques as presented in [2] and [5] combined with a modified 359 FFT algorithm for multistatic imaging with subsampled arrays 360 can be efficiently applied in this setup to reduce the number 361 of receivers in more than 90\% [2], although this analysis is 362 beyond the scope of this contribution. A $15-\mathrm{GHz}$ bandwidth 363 


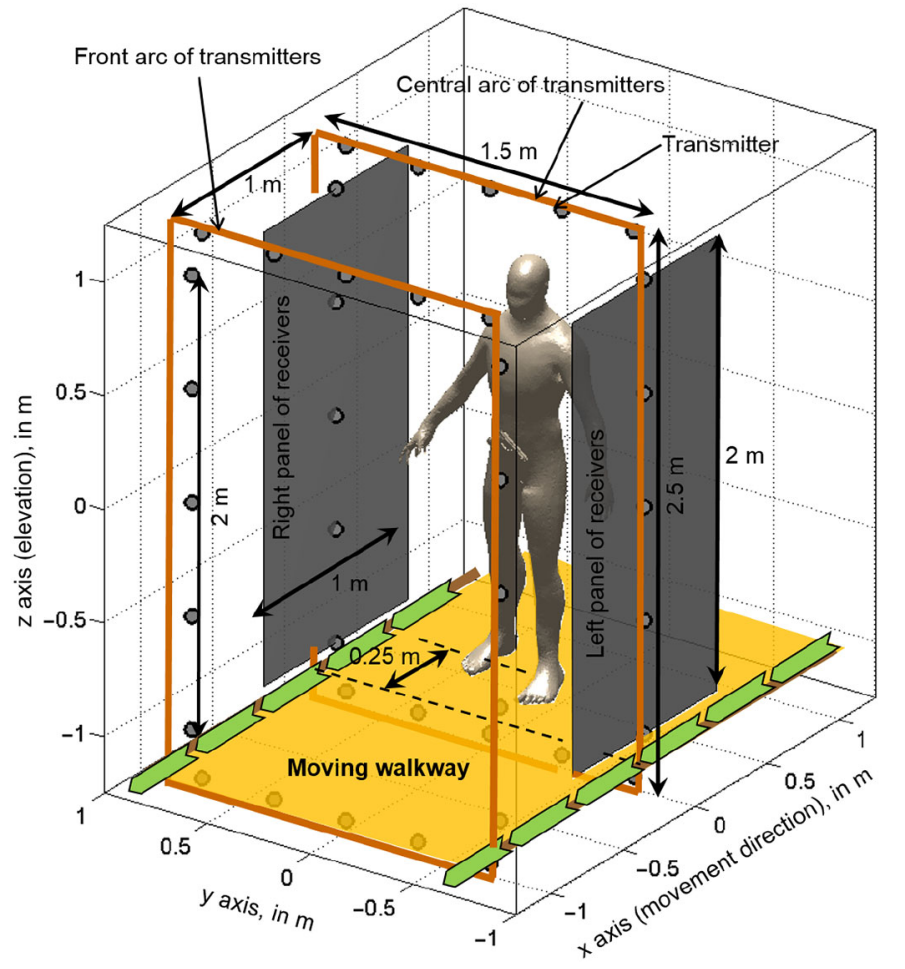

F12:1

F12:2

F12:3

Fig. 12. Layout of the mm-wave scanner for personnel screening. For the sake of simplicity, just two arcs of transmitters, at $\mathrm{x}=0 \mathrm{~m}$, and $\mathrm{x}=-1 \mathrm{~m}$, are considered. The person under test is placed at $\mathrm{x}=0.25 \mathrm{~m}$.

(BW), from 15 to $30 \mathrm{GHz}$, is chosen, similarly to the UWB imaging system described in [5]. This BW provides and approximate range resolution of $1 \mathrm{~cm}$, although, for near-field radar imaging, besides the frequency and aperture size, the final system lateral and range resolutions are given by (2) and (3) of [20], respectively.

Hallway scanner dimensions have been selected to provide a resolution similar to other mm-wave scanners, as shown in Table I. It must be reminded that the number of receiving elements can be reduced in the hallway system.

Concerning processing time, the fastest operational $\mathrm{mm}$ wave imaging systems listed in Table I are capable to provide detection results in less than $5 \mathrm{~s}$, so the scanning process can take up to $10 \mathrm{~s}$ taking into account that the person needs to be placed in a particular position within the scanner. For the presented system, the overall scanning process would be limited by the time the person needs to go through the hallway.

Three arcs of transmitters, centered at $\mathrm{x}=+1,0$, and $-1 \mathrm{~m}$, and each having 20 elements evenly spaced along $y$ - and z-axes, are considered. For the sake of simplicity, only the ones at -1 and $0 \mathrm{~m}$, depicted in Fig. 12, will be considered to obtain the results in this section. Some of the transmitters are placed on top and below the body to ensure the areas with larger curvature (as the top of the chest and shoulders) are reconstructed.

A physical optics (PO) code [21], [22] in combination with a visibility algorithm [23] has been used to predict the parts of the body model in Fig. 12 that are illuminated by every transmitter. Also, PO provides the amount of scattered field collected on the panels. Thus, it is possible to evaluate if a certain layout of transmitters is capable of illuminating the entire person after crossing the hallway and to estimate the field scattered by the (a)

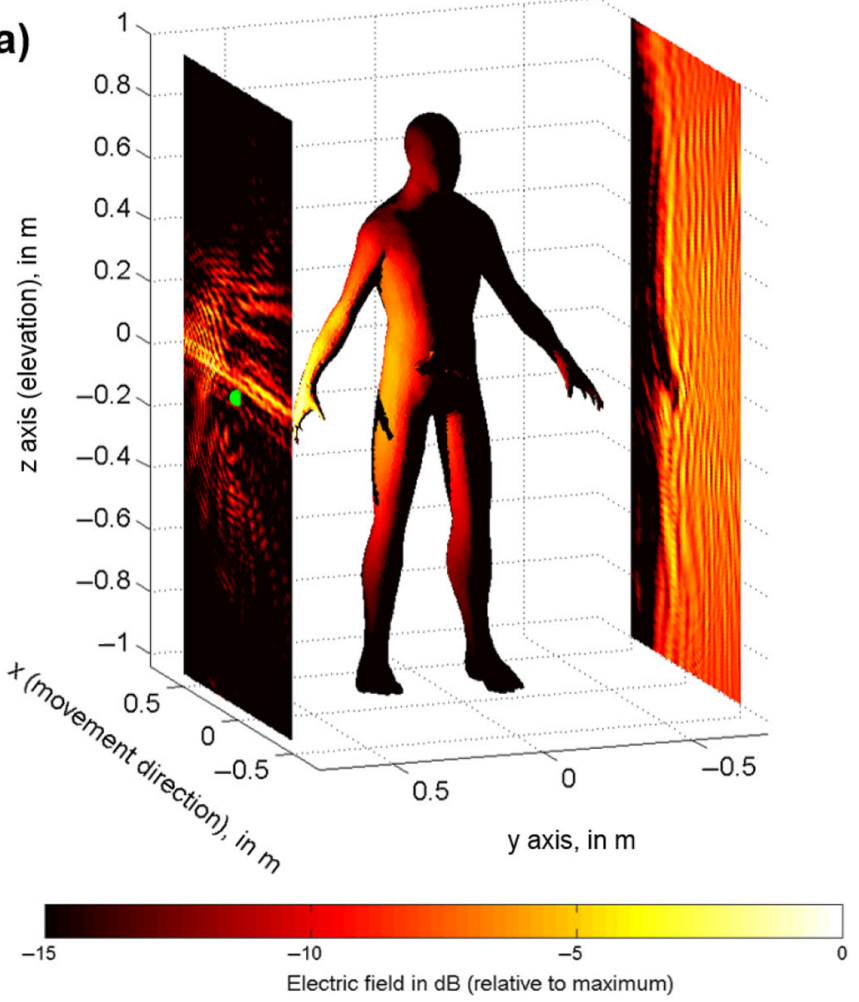

(b)

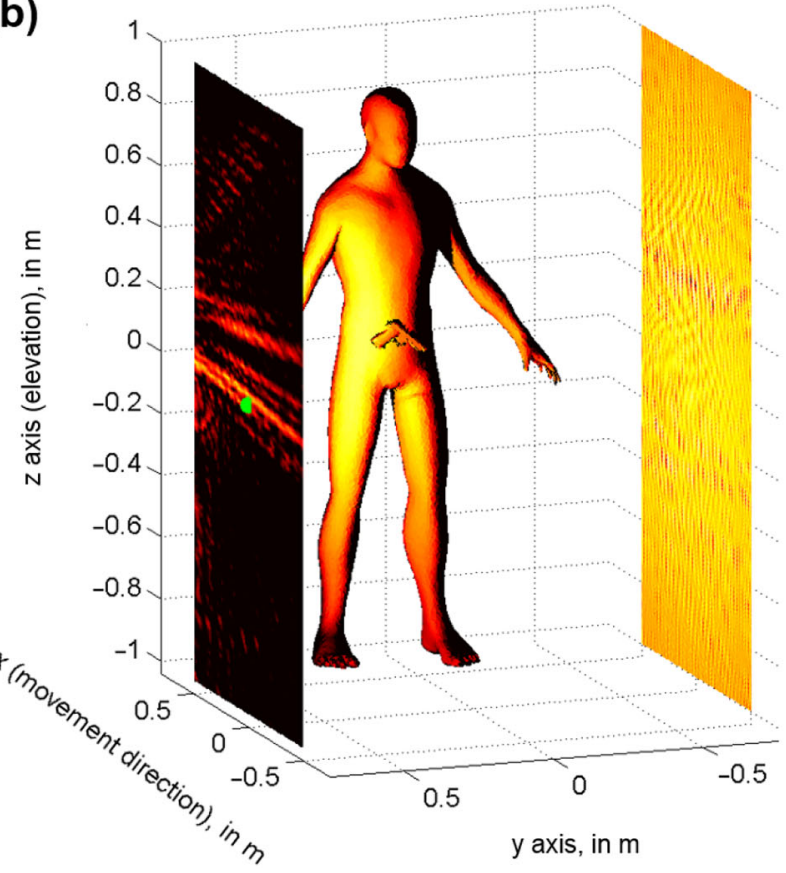

Fig. 13. Examples of human body illumination using one transmitter (high- F13:1 lighted in green) and scattered field on the array panels when the body model is F13:2 centered in (a) $0.25 \mathrm{~m}$ and (b) $0.75 \mathrm{~m}$.

F13:3

illuminated areas on the receiving panels. For these simulations, 395 the human body is assumed to behave as a PEC in the 15-30- 396 $\mathrm{GHz}$ frequency band.

As an example, Figs. 13 and 14 show the regions of the 398 human body under test illuminated by two different transmit- 399 ters, as well as the field received on the lateral panels. Note that, 400 even for a single position of the person in the hallway, different 401 

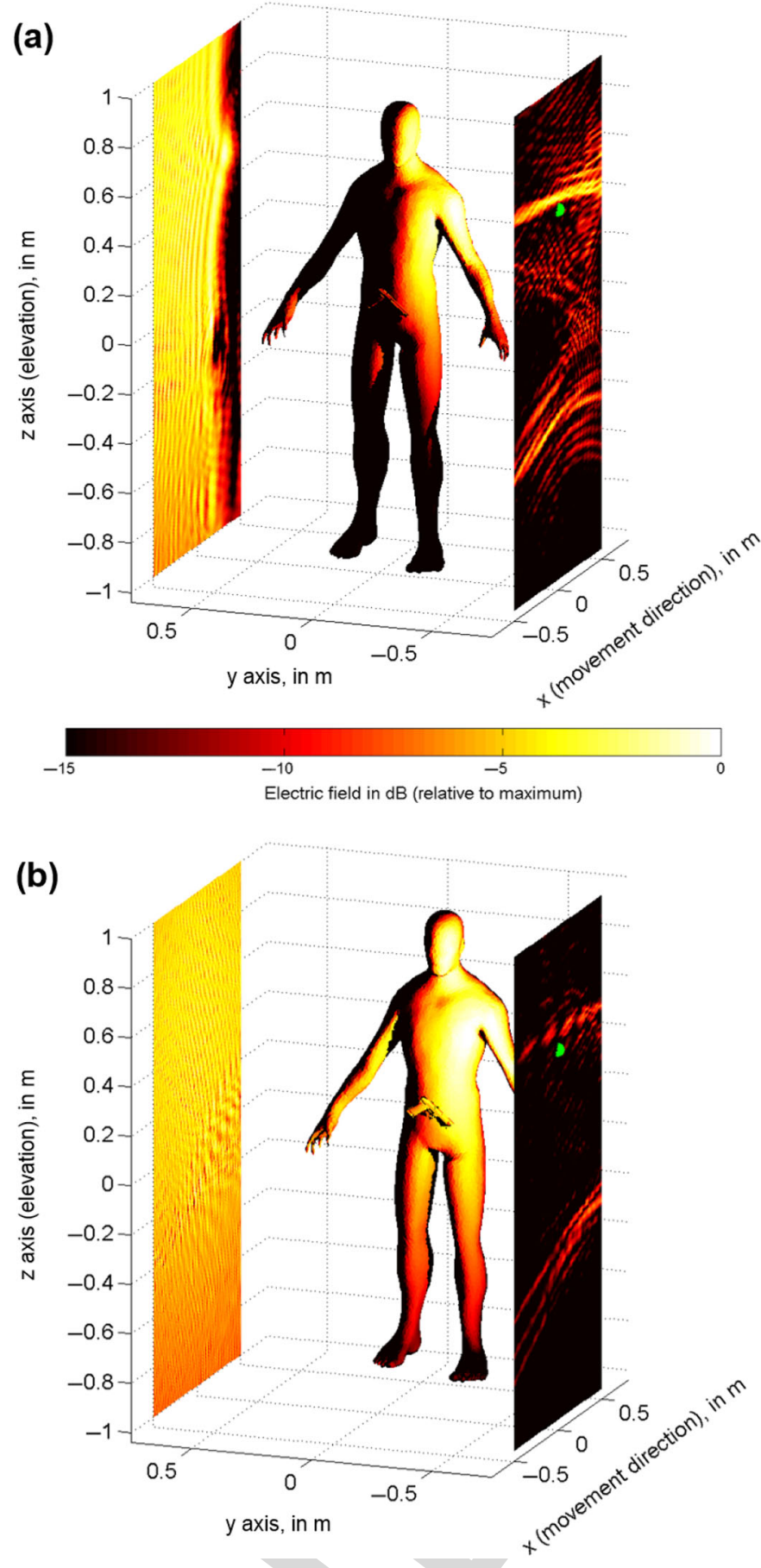

F14:1 Fig. 14. Examples of human body illumination using one transmitter (highF14:2 lighted in green) and scattered field on the array panels when the body model is F14:3 centered in (a) $0.25 \mathrm{~m}$ and (b) $0.75 \mathrm{~m}$. areas of the body are illuminated. This layout increases the amount of information thanks to the spatial diversity of the multistatic illumination.

Regarding the inverse method to create images in this system and due to the large computational cost for the imaging, when the backpropagation is implemented in 3-D, the abovementioned Fourier-based technique for multistatic imaging [9] has been used. The efficient use of fast Fourier transforms (FFT) provides 3-D whole body imaging in almost real time using conventional hardware.

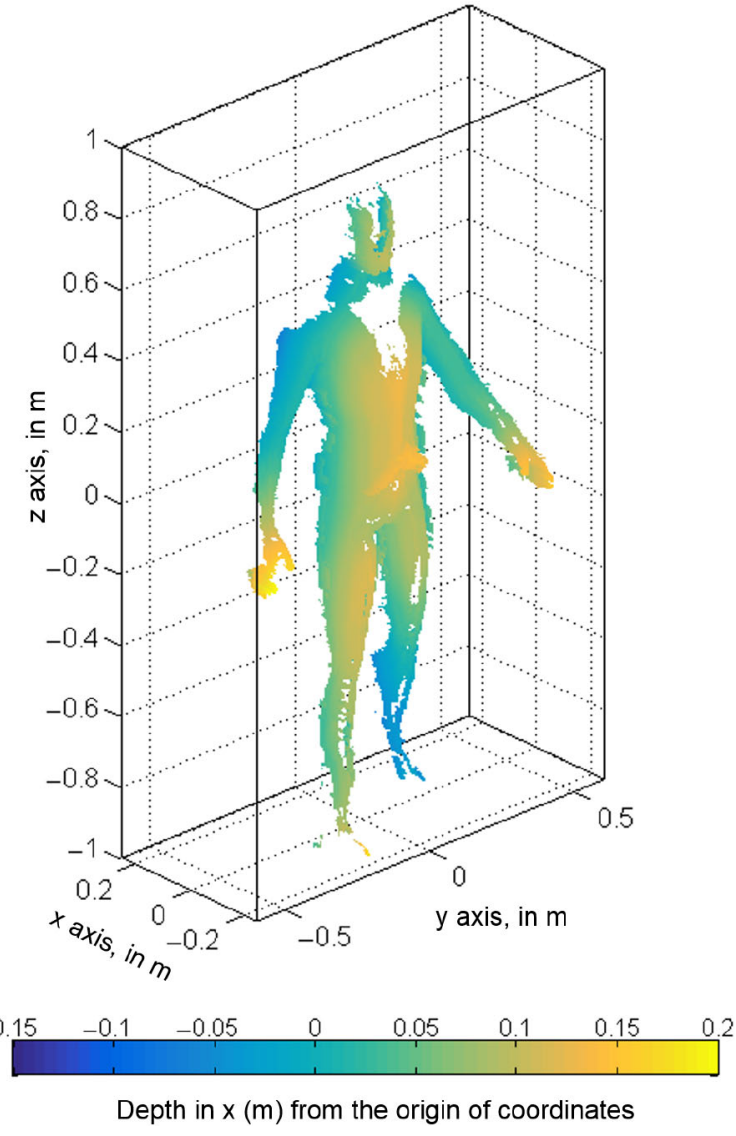

Fig. 15. Person placed at $x=0.25 \mathrm{~m}$. Recovered human body and concealed F15:1 object geometry from backpropagation imaging.

F15:2

As an application example to show the performance of the 412 proposed configuration, an OUT consisting on a person carry- 413 ing a concealed weapon in the belt has been considered. For 414 the sake of simplicity, only two positions are analyzed: person 415 standing at $x=0.25 \mathrm{~m}$ and at $\mathrm{x}=0.75 \mathrm{~m}$. In this example, the 416 goal is to clearly illustrate the different nature of the multistatic 417 information collected on each position, rather than a rigorous 418 reconstruction of the whole body.

For every position, transmitter, and receiving panel, the 420 amount of data to be processed is: $201 \times 401$ spatial samples $\times 421$ 121 frequency samples $\left(=9.75 \times 10^{6}\right.$ scattered field samples $), 422$ which also determines the number of imaging points in the 423 case of Fourier-based imaging [9]. A workstation with 32 cores 424 at $2.1 \mathrm{GHz}$ and 128-GB RAM was used for data processing. 425 Overall calculation time for every transmitter was $30 \mathrm{~s}(1200 \mathrm{~s} \quad 426$ total for the 40 used transmitters). The processing has been 427 done using a sequential Matlab code and has not been optimized 428 for real time imaging yet.

Imaging results are depicted in Figs. 15 and 16, correspond- 430 ing to the person's placement at $\mathrm{x}=0.25 \mathrm{~m}$ and $\mathrm{x}=0.75 \mathrm{~m}, 431$ respectively. Reflectivity points above $-25 \mathrm{~dB}$ with respect to 432 the maximum are coded in depth according to $\mathrm{x}$-axis, allowing 433 the recovery of the human body profile and potential concealed 434 weapons. Comparison of Figs. 15 and 16 provides a clear exam- 435 ple of the on-the-move imaging concept effectiveness. In the 436 case of Fig. 15 (person placed at $x=0.25 \mathrm{~m}$ ), the human body 437 sides and some areas of the chest are imaged by the system. In 438 


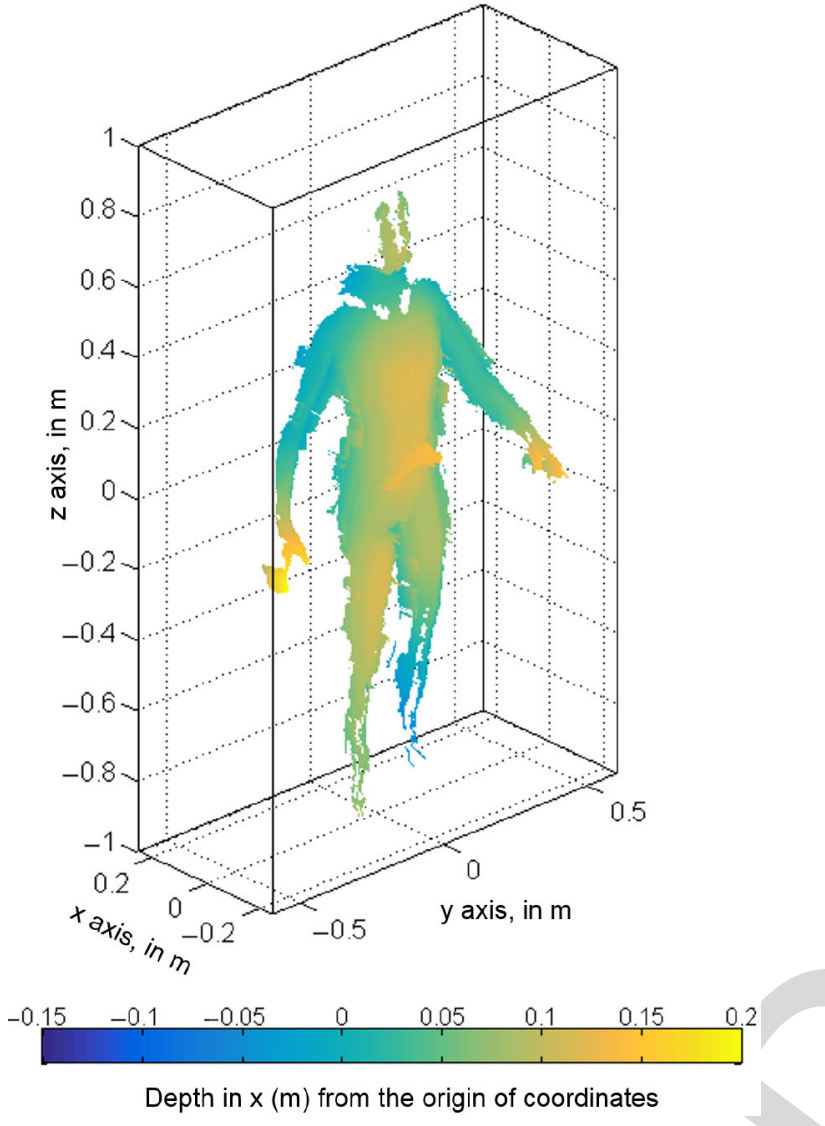

F16:1 Fig. 16. Person placed at $\mathrm{x}=0.75 \mathrm{~m}$. Recovered human body and concealed F16:2 object geometry from backpropagation imaging.
Fig. 16 (person placed at $x=0.75 \mathrm{~m}$ ), the top of the chest and the shoulders are recovered.

In the final system, multiple images, as the two presented examples, can be created and analyzed at video rate to detect any possible threats. Algorithms for mesh generation and automatic thread detection, such as the one used in [8], can be applied.

\section{CONCLUSION}

This work presented a novel concept for personnel scanning in airports and other checkpoints. Unlike the current imaging systems, the proposed system allows for continuous movement of the subject while being scanned; this will greatly increase the system throughput when compared with state-of-the-art systems. This improvement is possible thanks to the use of a fully multistatic radar configuration, where some of the transmitters and receivers are separated with a subtended angle relative to the person greater than 90 degrees to capture information from all possible wave incident angles. In this way, the system is able to create a complete contour reconstruction as the person moves inside the system. The use of a small number of transmitters allows for fast image creation as all the transmitters can be sequentially activated in a short amount of time. 2-D and 3-D simulation-based results confirm the good imaging capabilities of the proposed system; 2-D results have also been validated using measurements. Further work will be related with the setup optimization, including the use of sparse arrays and other tech- 464 niques to reduce the number of receivers, and with experimental 465 validation.

\section{REFERENCES}

[1] IATA. Checkpoint of the Future. Executive Summary [Online]. Available: http://www.iata.org/whatwedo/security/Documents/cof-executivesummary.pdf, accessed on Mar. 16, 2015.

[2] S. S. Ahmed, A. Schiessl, F. Gumbmann, M. Tiebout, S. Methfessel, and L. Schmidt, "Advanced microwave imaging," IEEE Microw. Mag., vol. 13, no. 6, pp. 26-43, Sep./Oct. 2012.

[3] S. S. Ahmed, "Personnel screening with advanced multistatic imaging technology," in Proc. SPIE Defense Secur. Sens., 2013, p. 87150B.

D. Sheen, D. McMakin, and T. Hall, "Three-dimensional millimeter-wave 476 imaging for concealed weapon detection," IEEE Trans. Microw. Theory 477 Techn., vol. 49, no. 9, pp. 1581-1592, Sep. 2001.

468 469 470Q2

471

5] X. Zhuge and A. Yarovoy, "A sparse aperture MIMO-SAR-based UWB 479 imaging system for concealed weapon detection," IEEE Trans. Geosci. 480 Remote Sens., vol. 49, no. 1, pp. 509-518, Jan. 2011.

[6] D. M. Sheen, D. L. McMakin, and T. E. Hall, "Combined illumination cylindrical millimeter-wave imaging technique for concealed weapon detection," in Proc. AeroSense, 2000, pp. 52-60. J. A. Martinez, F. Las-Heras, and C. M. Rappaport, "On the use of 486 compressed sensing techniques for improving multistatic millimeter- 487 wave portal-based personnel screening," IEEE Trans. Antennas Propag., 488 vol. 62, no. 1, pp. 494-499, Jan. 2014.

[8] B. Gonzalez-Valdes, Y. Alvarez-Lopez, J. A Martinez-Lorenzo, F. Las 490 Heras Andres, and C. M. Rappaport, "On the use of improved imag- 491 ing techniques for the development of a multistatic three-dimensional 492 millimeter-wave portal for personnel screening," Prog. Electromagn. 493 Res., vol. 138, pp. 83-98, 2013.

[9] Y. Alvarez et al., "Fourier-based imaging for multistatic radar systems," IEEE Trans. Microw. Theory Techn., vol. 62, no. 8, pp. 1798-1810, Aug. 2014.

[10] G. Yates, A. Horne, A. Blake, and R. Middleton, "Bistatic SAR image formation," Inst. Elect. Eng. Proc. Radar Sonar Navigat., vol. 153, no. 3 , pp. 208-213, Jun. 2006.

[11] R. Burkholder, I. Gupta, and J. Johnson, "Comparison of monostatic and bistatic radar images," IEEE Trans. Antennas Propag. Mag., vol. 45, no. 3 , pp. 41-50, Jun. 2003.

[12] B. Gonzalez-Valdes, C. Rappaport, and J. A. Lorenzo-Martinez, "Onthe-move active millimeter wave interrogation system using a hallway of multiple transmitters and receivers," in Proc. IEEE Antennas Propag. Soc. Int. Symp. (APSURSI), 2014, pp. 1107-1108. move millimiter wave interrogation system with a hallway of multiple transmitters and receivers," U.S. Patent 14562 094, Dec. 5, 2014.

14] M. Soumekh, "Bistatic synthetic aperture radar inversion with application 511 in dynamic object imaging," IEEE Trans. Signal Process., vol. 39, no. 9, 512 pp. 2044-2055, Sep. 1991.

[15] Y. Alvarez, J. Martinez, F. Las-Heras, and C. Rappaport, "An inverse fast multipole method for imaging applications," IEEE Antennas Wireless

[16] D. Andreuccetti, R. Fossi, and C. Petrucci, "An Internet resource for the calculation of the dielectric properties of body tissues in the frequency range $10 \mathrm{~Hz}-100 \mathrm{GHz}$," Internet document, 1997 [Online]. Available: http://niremf.ifac.cnr.it/tissprop/, accessed on Sep. 15, 2015, IFAC-CNR, Florence, Italy, 1997, based on data published by C. Gabriel et al. in 1996.

[17] A. W. Morgenthaler and C. M. Rappaport, "Scattering from lossy dielectric objects buried beneath randomly rough ground: Validating the semianalytic mode matching algorithm with 2-D FDFD," IEEE Trans. Geosci. Remote Sens., vol. 39, no. 11, pp. 2421-2428, Nov. 2001.

$18]$ C. M. Rappaport, Q. Dong, E. Bishop, A. Morgenthaler, and 526 M. E. Kilmer, "Finite difference frequency domain (FDFD) modeling of 527 two dimensional TE wave propagation," in Proc. URSI Symp. Conf., Pisa, 528 Italy, 2004.

[19] A. Arboleya, Y. Alvarez, and F. Las-Heras, "Millimeter and submillimeter planar measurement setup," in Proc. IEEE Antennas Propag. Soc. Int. Symp. (APSURSI), 2013, pp. 1-2.

[20] S. S. Ahmed, A. Schiessl, and L.-P. Schmidt, "A novel active real-time digital-beamforming imager for personnel screening," in Proc. 9th Eur. Conf. Synth. Aperture Radar (EUSAR), Apr. 2012, pp. 178-181.
481

482 5 Propag. Lett., vol. 10, pp. 1259-1262, Nov. 2011. 
[21] J. Meana, J. Martinez-Lorenzo, F. Las-Heras, and C. Rappaport, "Wave scattering by dielectric and lossy materials using the modified equivalent current approximation (MECA)," IEEE Trans. Antennas Propag., vol. 58, no. 11, pp. 3757-3761, Nov. 2010.

[22] L. E. Tirado, J. A. Martinez-Lorenzo, B. Gonzalez-Valdes, C. Rappaport, O. Rubinos-Lopez, and H. Gomez-Sousa, "GPU implementation of the modified equivalent current approximation (MECA) method," Appl. Comput. Electromagn. Soc. J., no. 9, Sep. 2012.

[23] J. Gutiérrez Meana, F. L. Las Heras Andrés, and J. Á. Martínez Lorenzo, "A comparison among fast visibility algorithms applied to computational electromagnetics," Appl. Comput. Electromagn. Soc. J., 2009.

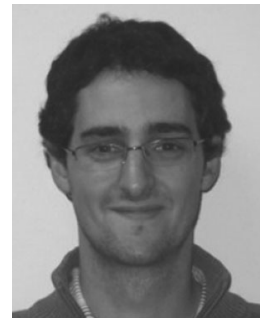

Borja Gonzalez-Valdes (M'xx) received the B.S and $\mathrm{Ph} . \mathrm{D}$. degrees in electrical engineering from the University of Vigo, Vigo, Spain, in 2006 and 2010, respectively.

From 2006 to 2010, he was with the Antenna and Optical Communications Group, University of Vigo. From 2008 to 2009, he was a Visiting Researcher with the Gordon Center for Subsurface Sensing \& Imaging Systems, Northeastern University, Boston, MA, USA. In 2011, he joined the Awareness and Localization of Explosives-Related Threats Center of Excellence, Northeastern University. Since 2015, he has been a Postdoctoral Researcher affiliated with the AtlantTIC Research Center, University of Vigo. His research interests include antenna design, inverse scattering, radar, advanced imaging techniques, and $\mathrm{THz}$ technology.

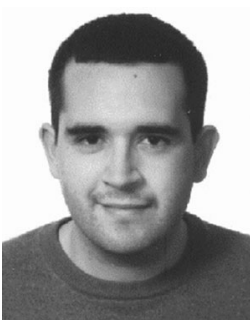

Yuri Álvarez (S'06-M'09-SM'15) was born in Langreo, Spain, in 1983. He received the M.S. and $\mathrm{Ph} . \mathrm{D}$. degrees in telecommunication engineering from the University of Oviedo, Gijn, Spain, in 2006 and 2009, respectively.

He was a Visiting Scholar at the Department of Electrical Engineering and Computer Science, Syracuse University, Syracuse, NY, USA, in 2006 and 2008; a Visiting Postdoc at the Gordon Center for Subsurface Sensing and Imaging Systems (CenSSIS)ALERT (Awareness and Localization of Explosive Related Threats) Center of Excellence, Northeastern University, Boston, MA, USA, from 2011 to 2014; and a Visiting Postdoc at ELEDIA Research Center, Trento, Italy, in 2015. He is currently an Assistant Professor with the Signal Theory and Communications, University of Oviedo, Gijn, Spain. His research interests include antenna diagnostics, antenna measurement techniques, RF techniques for indoor location, inverse scattering and imaging techniques, and phaseless methods for antenna diagnostics and imaging.

Dr. Alvarez was the recipient of the 2011 Regional and National Awards to the Best Ph.D. Thesis on Telecommunication Engineering (category: security and defense).

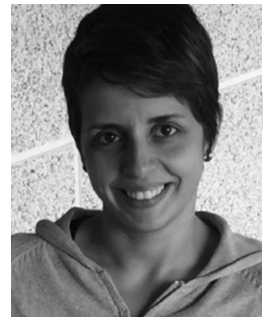

Yolanda Rodriguez-Vaqueiro ( $S^{\prime} x x$ ) received the B.S. and M.S. degrees in electrical engineering from the University of Vigo, Vigo, Spain, in 2009, and the $\mathrm{Ph} . \mathrm{D}$. degree in electrical engineering from Northeastern University, Boston, MA, USA, in 2015 (after defending her thesis: Compressive Sensing for Electromagnetic Imaging Using a Nesterov-Based Algorithm).

She is a Postdoctoral Researcher affiliated with the AtlantTIC Research Center, University of Vigo. In 2011, she obtained a Research Assistant grant from the ALERT (Awareness and Localization of Explosive Related Threats) Center of Excellence, Northeastern University. She was also granted as a Junior Researcher with the University of Vigo.

Dr. Rodriguez-Vaqueiro was the recipient of the Research-Impact Award by the Department of Electrical and Computer Engineering, Northeastern University (for her work during the Ph.D. studies), the Best Paper Award in the 2012 IEEE Homeland Security Conference, Honorable Mention in the Student Paper Competition in the 2013 IEEE APS/URSI Conference, the Best Paper Award in the 2014 European Conference on Antennas and Propagation, the Burke/Yannas Award to the most original research study in the field of bioengineering in the 2015 American Burn Association (ABA) Meeting, and the Research-Impact Award by the Department of Electrical and Computer Engineering, Northeastern University, in May 2015.

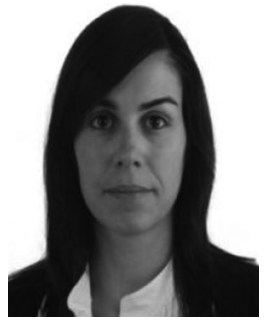

Ana Arboleya-Arboleya received the M.Sc. 607 degree in telecommunication engineering from the 608 University of Oviedo, Oviedo, Spain, in 2009, where 609 she is currently pursuing the Ph.D. degree in telecom- 610 munication engineering. Since 2008, she has been 611 a Research Assistant within the Signal Theory and 612 Communications Research Group, TSC-UNIOVI, 613 Department of Electrical Engineering, University of 614 Oviedo. She was a Visiting Scholar in 2014 and 2015615 at the Department of Radio Science and Engineering 616 and MilliLab, Aalto University, Espoo, Finland. Her 617 research interests include antenna diagnostics and measurement systems and 618 techniques and high-frequency imaging techniques and applications.

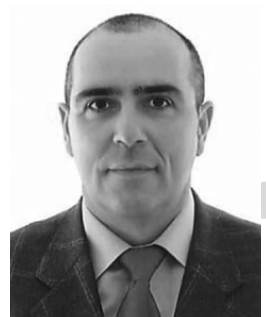

Antonio García-Pino (S'87-M'89-SM'05) was 620 born in Valdemoro, Madrid, Spain, in 1962. He 621 received the M.S. and Ph.D. degrees in telecommuni- 622 cations engineering from the Polytechnic University 623 of Madrid (UPM), Madrid, Spain, in 1985 and 1989, 624 respectively.

From 1985 to 1989, he was a Research Assistant 626 with the Radiation Group, UPM. He joined as 627 an Associate Professor with the Department of 628 Technologies of Communications, University of 629 Vigo, Vigo, Spain, in 1989, becoming Full Professor 630 in 1994. In 1993, he was a Visiting Researcher at the Center for 631 Electromagnetics Research, Northeastem University, Boston, MA, USA. From 632 2006 to 2010, he was the Vice-Rector of Academic Organization and Faculty, 633 and currently, he is the Director of the International Doctoral School, both at 634 University of Vigo. His research interests include shaped-reflector antennas for 635 communication and radar applications, high-frequency backscattering, compu- 636 tational electromagnetics, and THz technology. In these topics, he has authored 637 more than 100 technical papers in journal and conferences and he has been an 638 advisor of $14 \mathrm{Ph} . \mathrm{D}$. thesis.

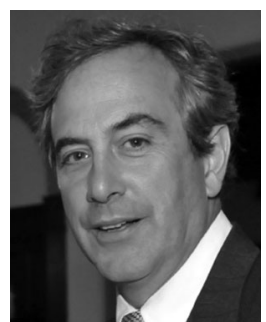

Carey M. Rappaport (SM'96-F'06) received the 640 S.B. degree in mathematics, the S.B., S.M., and E.E. degrees in electrical engineering in 1982, and the $\mathrm{Ph} . \mathrm{D}$. degree in electrical engineering in 1987 from the Massachusetts Institute of Technology (MIT), Cambridge, MA, USA.

He was a Teaching and Research Assistant with MIT from 1981 until 1987, and during the sum- 647 mers at COMSAT Labs, Clarksburg, MD, USA, 648 and the Aerospace Corp., El Segundo, CA, USA. 649 $\mathrm{He}$ joined the faculty at Northeastern University, 650 Boston, MA, USA, in 1987. He has been a Professor of Electrical and 651 Computer Engineering since July 2000. In 2011, he was appointed as a 652 College of Engineering Distinguished Professor. In fall 1995, he was a 653 Visiting Professor of Electrical Engineering at the Electromagnetics Institute, 654 Technical University of Denmark, Lyngby, Denmark, as part of the W. Fulbright 655 International Scholar Program. In the second half of 2005, he was a Visiting 656 Research Scientist at the Commonwealth Scientific Industrial and Research 657 Organisation (CSIRO), Epping, Australia. He has consulted for CACI, Alion 658 Science and Technology, Inc., Geo-Centers, Inc., PPG, Inc., and several munic- 659 ipalities on wave propagation and modeling, and microwave heating and safety. 660 He was the Principal Investigator of an ARO-sponsored Multidisciplinary 661 University Research Initiative on Humanitarian Demining, the Co-Principal 662 Investigator of the NSF-sponsored Engineering Research Center for Subsurface 663 Sensing and Imaging Systems (CenSSIS), and the Co-Principal Investigator 664 and Deputy Director of the DHS-sponsored Awareness and Localization of 665 Explosive Related Threats (ALERT) Center of Excellence. He has authored 666 more than 400 technical journal and conference papers in the areas of 667 microwave antenna design, electromagnetic wave propagation and scattering 668 computation, and bioelectromagnetics, and has received two reflector antenna 669 patents, two biomedical device patents, and four subsurface sensing device 670 patents.

Prof. Rappaport is a member of Sigma Xi and Eta Kappa Nu professional 672 honorary societies. He was the recipient of the IEEE Antenna and Propagation 673 Society's H. A. Wheeler Award for the Best Applications Paper, as a Student in 674 1986. (n) (1)

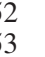

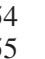
(n) (n) (n)

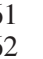
(n)

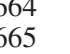
(n) (n) 
676

677

678

679

680

681

682

683

684

685

686

687

688

689

690

691

692

693

694

695

696

697

698

699

700

701

702

703

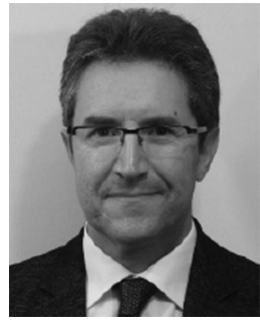

Fernando Las-Heras (M'86-SM'08) received the M.S. and Ph.D. degrees in telecommunication engineering from the Technical University of Madrid (UPM), Madrid, Spain, in 1987 and 1990, respectively.

He was a National Graduate Research Fellow (1988-1990), and he held a position of Associate Professor with the Department of Signal, Systems, and Radiocommunications, UPM (1991-2000). From December 2003, he holds a Full Professor position with the University of Oviedo, Oviedo, Spain, where he was the Vice-Dean for Telecommunication Engineering, Technical School of Engineering, Gijón, Spain (2004-2008). As of 2001, he was the Head of the Research Group Signal Theory and Communications TSC-UNIOVI, Department of Electrical Engineering, University of Oviedo. He was a Visiting Lecturer at the National University of Engineering, Rímac Lima, Peru, in 1996, a Visiting Researcher at Syracuse University, Syracuse, NY, USA, in 2000, and a short-term Visiting Lecturer at ESIGELEC, France, from 2005 to 2011. He held the Telefónica Chair on RF Technologies, ICTs applied to Environment and ICTs and Smartcities with the University of Oviedo (2005-2015). He has authored more than 300 articles published in academic journals and proceedings of international conferences, mainly in the areas of antenna design and the inverse electromagnetic problem with applications in diagnostic, measurement and synthesis of antennas, phaseless techniques, propagation, and microwave to $\mathrm{THz}$ imaging and localization, as well as in engineering education.

Dr. Las-Heras was a Member of the Board of Directors of the IEEE Spain Section (2012-2015), and from 2010, he was a Member of the Science, Technology, and Innovation Council of Asturias, Asturias, Spain.

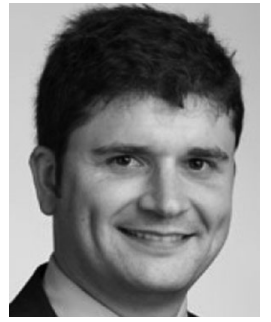

Jose A. Martinez-Lorenzo (M'xx) received the 704 B.S./M.S. degree in 2002 and the Ph.D. degree in 705 2005 from the University of Vigo, Vigo, Spain, both 706 in electrical engineering.

$\mathrm{He}$ joined the faculty at University of Oviedo, 708 Gijon, Spain, in 2004, where he was an Assistant 709 Professor with the Department of Signal Theory 710 and Communications. In 2006, he joined Bernard 711 M. Gordon Center for Subsurface Sensing and 712 Imaging Systems, Northeastern University, Boston, 713 MA, USA. In 2010, he was a Research Assistant 714 Professor with the Department of ECE, Northeastern University. Since August 715 2013, he has been held a joint appointment with the Departments of MIE 716 and ECE as a Tenure-Track Assistant Professor. He is an Active Member of 717 Awareness and Localization of Explosives-Related Threats (ALERT) a DHS 718 Center of Excellence awarded to Northeastern University. He has authored 719 more than 140 technical journal and conference papers. His research inter- 720 ests include the understanding, modeling, and solving complex engineering 721 problems, with an emphasis on mechanical and electromagnetic sensing and 722 imaging methods for security and biomedical applications (i.e., explosive 723 detection, breast cancer detection).

Prof. Martinez-Lorenzo has received funding from multiple agencies, includ- 725 ing: DHS, DARPA, NSF, US Army, and the European Space Agency (ESA). He 726 led the team that won the Best Paper Award in the 2012 IEEE Conference on 727 Technologies for Homeland Security, for the paper on a compressed sensing 728 approach for detection of explosive threats at standoff distances using a passive 729 array of scatterers. 


\section{QUERIES}

Q1: Please provide captions for Fig. 3 subparts.

Q2: Please provide year of publication for Ref. [1].

Q3: Please provide page range for Refs. [18], [22], and [23].

Q4: Please provide volume number for Ref. [22] and [23].

Q5: Please provide the membership history (year) of the authors Borja Gonzalez-Valdes, Yolanda Rodriguez-Vaqueiro,and Jose A. Martinez-Lorenzo.

Q6: Please provide year of completion for the S.B. degree in Mathematics, S.B., S.M. degrees in electrical engineering of author "Carey M. Rappaport." 


\title{
Millimeter Wave Imaging Architecture for On-The-Move Whole Body Imaging
}

\author{
Borja Gonzalez-Valdes, Member, IEEE, Yuri Álvarez, Senior Member, IEEE, \\ Yolanda Rodriguez-Vaqueiro, Student Member, IEEE, Ana Arboleya-Arboleya, \\ Antonio García-Pino, Senior Member, IEEE, Carey M. Rappaport, Fellow, IEEE, \\ Fernando Las-Heras, Senior Member, IEEE, and Jose A. Martinez-Lorenzo, Member, IEEE
}

Abstract-This paper presents a novel interrogation system that combines multiple millimeter wave transmitters and receivers to create real-time high-resolution radar images for personnel security screening. The main novelty of the presented system is that the images can be created as the person being screened continuously moves across a corridor where the transmitters and receivers, working in a fully coherent architecture, are distributed. As the person moves, the transmitters and receivers are sequentially activated to collect data from different angles to inspect the whole body. Multiple images, similar to video frames, are created and examined to look for possible anomalies such as concealed threats. Two-dimensional (2-D) and three-dimensional (3-D) setups have been simulated to show the feasibility of the proposed system. The simulation results in 2 -D have been validated using measurements.

Index Terms-Backpropagation imaging, checkpoint, fast Fourier transform (FFT), imaging systems, multistatic radar system.

\section{INTRODUCTION}

$\mathbf{I}$ $\mathrm{N}$ homeland security applications, there is an increasing demand for methods to improve personnel screening for concealed object and contraband detection at security checkpoints. In this context, active nearfield millimeter-wave (mm-wave) imaging radar systems are able to provide highresolution imaging at an affordable cost. The object of interest is first illuminated by mm waves and then the scattered field is

Manuscript received June 18, 2015; revised January 11, 2016; accepted February 18, 2016. Date of publication XXXX XX, XXXX; date of current version XXXX XX, XXXX. This work was supported in part by the Ministerio de Ciencia e Innovacin of Spain/FEDER under project MIRIIEM-TEC201454005-P, in part by the Gobierno del Principado de Asturias through the PCTI 2013-2017, GRUPIN14-114, in part by the Spanish Government under project TACTICA, in part by the European Regional Development Fund (ERDF), in part by the Galician Regional Government under Projects CN2012/279, CN2012/260 (AtlantTIC) and the Plan I2C (2011-2015), and in part by the Science and Technology Directorate, U.S. Department of Homeland Security under the Award Number 2008-ST-061-ED0001.

B. Gonzalez-Valdes, Y. Rodriguez-Vaqueiro, and A. García-Pino are with the Atlantic Research Center, Universidad de Vigo, 36310 Vigo, Spain (e-mail: bgvaldes@com.uvigo.es; yrvaqueiro@com.uvigo.es; agpino@com.uvigo.es).

Y. Álvarez, A. Arboleya Arboleya, and F. Las-Heras are with the Area of Signal Theory and Communications, Department of Electrical Engineering, Universidad de Oviedo, E-33203 Gijón, Spain (e-mail: yalopez@tsc.uniovi.es; aarboleya@tsc.uniovi.es; flasheras@tsc.uniovi.es).

C. M. Rappaport and J. A. Martinez are with the ALERT Center, Northeastern University, Boston, MA 02115 USA (e-mail: rappapor@ece.neu.edu; jmartine@ece.neu.edu).

Color versions of one or more of the figures in this paper are available online at http://ieeexplore.iee.org.

Digital Object Identifier 10.1109/TAP.2016.2539372 measured and processed to reconstruct the surface (or volume) of the object.

The development of checkpoints that allow high passenger flow is becoming a priority. This has motivated the design of mm-wave imaging systems that minimize passenger inconvenience.

The International Air Transport Association (IATA) has defined several specifications that future checkpoints for personnel screening should meet. Novel paradigms in the design of the checkpoints specify that "from 2020 and beyond it is envisaged that the passenger will be able to flow through the security checkpoint without interruption unless the advanced technology identifies a potential threat," [1] (page 14).

In [1], a computer graphics design of the checkpoint of the future proposed by IATA is presented. The novelty with respect to existing architectures is the inclusion of a beltway or hallway to avoid passenger flow interruption.

Current state-of-the-art mm-wave imaging systems for security screening require people to enter and stand in front of the scanning system. Mm-wave generation and acquisition can be achieved using static arrays of transmitters and receivers [2], [3], or using movable arrays to create planar [4], [5], or cylindrical [6]-[8] acquisition domains. Most of them are based on monostatic radar and Fourier inversion [2]-[6]. Monostatic imaging systems are cost effective, but they are only able to reconstruct surfaces that create specular reflection and they are not well suited for imaging scattering objects with sudden profile variations [9]. Further, they are prone to dihedral artifacts as described in [8], [10], and [11].

Based on the new checkpoint architecture proposed by the IATA, this paper introduces a novel concept for mm-wave scanning system for personnel screening. The proposed imaging system does not include any mechanical movement, and whole body imaging is obtained taking advantage of the movement of the person under test when passing through the system on a moving walkway.

The main contribution of this paper is the introduction of this novel architecture, called on-the-move imaging [12], [13], that, to the best of the author's knowledge, has not been previously conceived nor demonstrated.

This paper is structured as follows. Section II describes the proposed mm-wave screening system. Imaging algorithm for multistatic setups is briefly described in Section III. Proof-ofconcept is validated through two-dimensional (2-D) simulation 

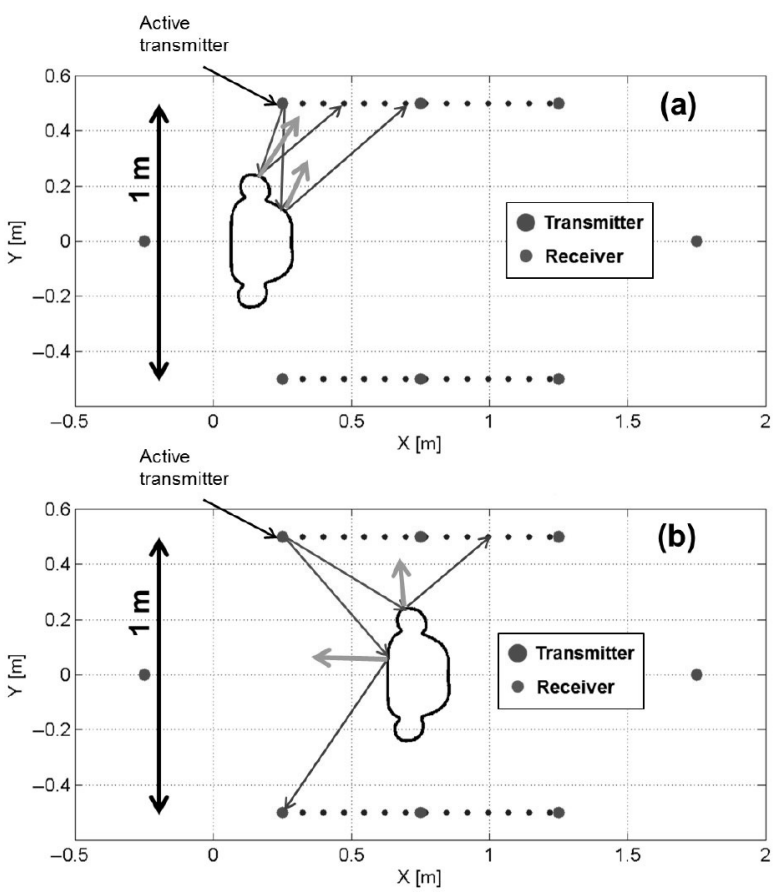

F1:1 Fig. 1. On-the-move imaging concept. OUT movement between the two walls F1:2 of radar antennas provides multiple points-of-view for every transmitter and $\mathrm{F} 1: 3$ receiver, thus increasing multistatic information. (a) and (b) represent two F1:4 different OUT positions within the hallway. examples in Section IV, and measurement results in Section V. Extension to two-dimensional (3-D) whole human body imaging is described and validated in Section VI. Finally, the conclusion is presented in Section VII.

\section{ON-ThE-Move Hallway CONCEPT}

The novel mm-wave on-the-move imaging system for personnel screening takes advantage of: 1) the movement of the person when passing through the imaging system and 2) a mulstitatic radar configuration, where some of the transmitters and receivers are separated with a subtended angle relative to the person equal or greater than $90^{\circ}$ to capture information from all possible wave incident and scattering angles.

A top view of the suggested multistatic architecture is plotted in Fig. 1. Several transmitters (red dots) and receivers (blue dots) are placed on the sides of the hallway. The person moves along the security checkpoint on a moving walkway.

The imaging radar system takes advantage of multiple incidence angles that illuminate different areas of the person depending on the active transmitter and the placement of the person within the hallway, as illustrated in Fig. 1. A single transmitter can illuminate different areas of the person while crossing the hallway. Reciprocally, the scattered field is collected by different receivers depending on the transmitting element and the current position of the person. This is illustrated with the red and blue arrows in Fig. 1 that represent direct reflection contributions given by the incident angle and the normal to the surface according to Snell's law.

Multistatic information can be incremented by placing transmitters at the hallway ends. For practical implementation, this
TABLE I

COMPARISON With StATE-OF-THE-ART MM-WAVE IMAGING SySTEMS

\begin{tabular}{|c|c|c|c|c|}
\hline Reference & $\begin{array}{l}\text { Scanning } \\
\text { area } \\
(\mathrm{cm})^{1}\end{array}$ & $\begin{array}{l}\text { PSF } \\
(\mathrm{mm})^{2}\end{array}$ & $\begin{array}{l}\text { Frequency } \\
\text { band } \\
(\mathrm{GHz})\end{array}$ & $\begin{array}{l}\text { Number of } \\
\text { antennas }\end{array}$ \\
\hline $\begin{array}{l}\text { On-the- } \\
\text { move }\end{array}$ & $100 \times 200^{3}$ & $10 \times 10$ & $15-30$ & $\begin{array}{l}2 \times 80601 \mathrm{Rx} \\
60 \mathrm{Tx}\end{array}$ \\
\hline $\begin{array}{l}\text { UWB MIMO } \\
\text { array, [5] }\end{array}$ & $50 \times 130$ & $10 \times 10$ & $2.8-19.5$ & $\begin{array}{l}4 \mathrm{Tx} \quad 8 \mathrm{Rx}, \\
\text { Height mo- } \\
\text { tion. }\end{array}$ \\
\hline $\begin{array}{l}\text { Flat 2-D array, } \\
\text { [2] }\end{array}$ & $100 \times 200$ & $3.0 \times 1.5$ & $72-80$ & $\begin{array}{ll}3072 & \mathrm{Tx} \\
3072 \mathrm{Rx} & \end{array}$ \\
\hline $\begin{array}{l}\text { Linear array, } \\
\text { vertical } \\
\text { movement } \\
{[4]}\end{array}$ & $\begin{array}{l}72.6 \\
\text { Movable } \\
2 \mathrm{~m} \text { in } \\
\text { height }\end{array}$ & $10.0 \times 3.8$ & $27-33$ & $\begin{array}{l}66 \mathrm{Tx}, \quad 66 \\
\mathrm{Rx}, \mathrm{Height} \\
\text { motion }\end{array}$ \\
\hline
\end{tabular}

${ }^{1}$ Scanning area size: width $\times$ height.

${ }^{2}$ PSF (point spread function): range $\times$ cross range.

${ }^{3}$ Receiving panels size.

would partially block the persons path. This is solved in the 3-D 105 case placing the receivers at the hallway ends below and above 106 the moving walkway.

For every transmitter, the scattered field is collected on the 108 receiving arrays placed on the hallway sides, and for every 109 receiving array, a reflectivity image is recovered. The reflectiv- 110 ity images associated with each transmitter are coherently com- 111 bined. This configuration assumes that, for a single position, 112 the body remains still while all the transmitters are sequentially 113 activated and the scattered field is collected by the receivers. 114 In this sense, and since the acquisition on the receivers can be 115 done in parallel, the use of a low number of transmitters is desir- 116 able. A fully electronic scanning system similar to the one in [3] 117 would easily allow for such an acquisition procedure.

A critical aspect in the design of the imaging system is the 119 selection of the frequency band. Table I shows a comparison 120 among the proposed hallway concept and some of the exist- 121 ing mm-wave scanning systems. It can be observed that, for 122 a given size of the scanner, higher frequency bands provide 123 better cross-range resolution, at the expense of losing dynamic 124 range due to free-space propagation losses. Furthermore, cloth- 125 ing becomes less transparent for these higher frequency bands, 126 and radiofrequency hardware becomes more expensive. The 127 work presented in [5] addresses the aforementioned drawbacks 128 introducing an ultra-wideband (UWB) imaging system. In addi- 129 tion to the improved range resolution and dynamic range, the 130 novelty of this study is the fact that the sampling rate can be 131 relaxed by taking advantage of grating lobes cancellation in 132 UWB arrays, which will be of interest concerning practical 133 implementation of the on-the-move architecture.

\section{IMAGING ALGORITHM}

Practical mm-wave scanning system implementation 136 demands real-time imaging capabilities. Standard backprop- 137 agation techniques [14] require millions of calculations for 138 electrically large acquisition and imaging domains. To illus- 139 trate the numerical magnitude of the problem, typical values 140 for acquisition points and imaging voxels are $10^{5}$ and $10^{7}, 141$ 
respectively, assuming an operational frequency of $30 \mathrm{GHz}$ $(\lambda=1 \mathrm{~cm})$ and sampling every half wavelength in both domains according to Nyquist criterion.

The reflectivity function on a volumetric domain $\rho_{t}\left(x^{\prime}, y^{\prime}, z^{\prime}\right)$ can be recovered from the scattered field $E_{\text {scatt }}^{t}(f, x, z)$ acquired on a flat receiving aperture placed at $y=Y_{0}$, by solving the following integral equation [9], [14], when the $t$ th (with $t$ from 1 to $N_{t x}$ ) of a group of transmitters is active

$$
\begin{aligned}
& \rho_{t}\left(x^{\prime}, y^{\prime}, z^{\prime}\right) \\
&= \iiint E_{\text {scatt }}^{t}(f, x, z) e^{+j k\left(\left(x-x^{\prime}\right)^{2}+\left(Y_{0}-y^{\prime}\right)^{2}+\left(z-z^{\prime}\right)^{2}\right)^{1 / 2}} \\
& e^{+j k\left(\left(x_{i n c}^{t}-x^{\prime}\right)^{2}+\left(y_{i n c}^{t}-y^{\prime}\right)^{2}+\left(z_{\text {inc }}^{t}-z^{\prime}\right)^{2}\right)^{1 / 2}} d f d x d z
\end{aligned}
$$

where $\left(x_{i n c}^{t}, y_{i n c}^{t}, z_{i n c}^{t}\right)$ denotes the position of the $t$ th point source-like transmitter, $k=2 \pi f / c$, y-axis is the range axis (depth), $\mathrm{x}$ - and $\mathrm{z}$-axes are horizontal and vertical cross ranges, and $f$ is the frequency.

Fast propagation techniques, such as the inverse fast multipole method, have been proposed [15], reducing the calculation time by several orders of magnitude. Moreover, (1) can be parallelized taking advantage of GPU hardware. However, these solutions are still too computationally expensive for applications requiring real-time imaging.

Fourier-based techniques have been widely used in monostatic setups for real-time imaging [3]-[5], thanks to the fact that plane wave incidence can be considered during the inversion. Multistatic setups require different Fourier processing as the transmitter and receiver are placed in different positions. A novel Fourier-based imaging technique, totally suitable for the proposed hallway-based on-the-move imaging system, is presented in [9]. The idea is to decompose the imaging domain in smaller regions where an incident spherical wave can be locally treated as a plane wave. Imaging calculations for every region can be carried out in parallel, without jeopardizing the required real-time capabilities of the multistatic imaging system.

When multiple transmitters are used, the final reconstruction for a certain voxel placed in $\left(x^{\prime}, y^{\prime}, z^{\prime}\right)$ can be obtained by combining the images generated by each transmitter as

$$
\rho\left(x^{\prime}, y^{\prime}, z^{\prime}\right)=\sum_{t=1}^{N_{t x}} \rho_{t}\left(x^{\prime}, y^{\prime}, z^{\prime}\right) \text {. }
$$

This formulation assumes all the transmitters and receivers work in a fully coherent configuration using a clock signal that provides common phase reference.

\section{2-D RESULTS}

The proposed on-the-move imaging is first validated using a 2-D example. The frequency band ranges from 15 to $30 \mathrm{GHz}$, sampled every $300-\mathrm{MHz}$ frequency steps and providing $1-\mathrm{cm}$ range resolution. Two 1-m width lateral arrays of receivers with 50 evenly spaced elements are placed at $Y_{0}=-0.6 \mathrm{~m}$ and $Y_{0}=0.6 \mathrm{~m}$. Five transmitters are interleaved among each

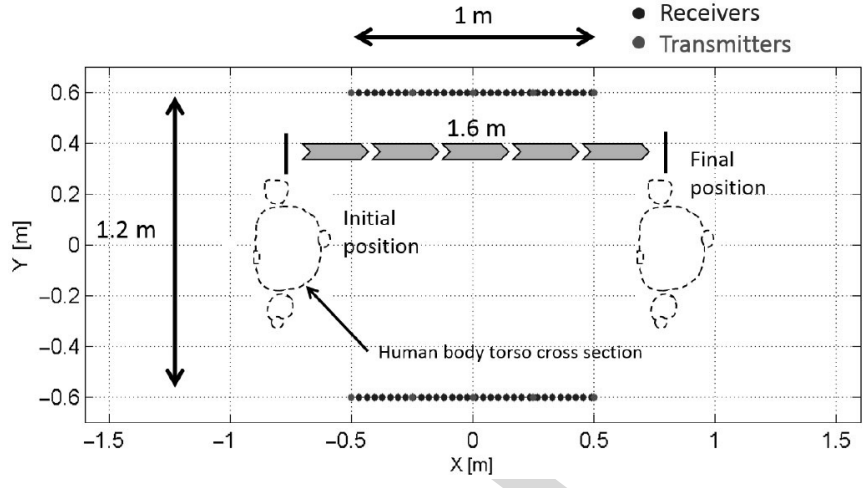

Fig. 2. 2-D example layout. OUT is displaced from position $x=-0.8 \mathrm{~m}$ to $\mathrm{F} 2: 1$ $x=+0.8 \mathrm{~m}$, in five steps $\left(N_{\text {pos }}=5\right) .5 \mathrm{Tx}$ and $50 \mathrm{Rx}$ per side are considered. F2:2

panel of receivers, thus resulting in $N_{t x}=10$ transmitters. The 186 described layout is plotted in Fig. 2. The essential aspect is that, 187 in order to image the entire body surface, for every transmitter, 188 receivers on both walls must receive the scattered waves (not 189 just those adjacent to a given transmitter.)

The object under test (OUT) models the cross section of 191 a human body torso (for a more realistic simulation, arms, 192 and waist are not connected), with three attached objects on 193 it represented as protrusions on the front, back, and arm. The 194 object in the front is an elliptical cross-sectional metallic object. 195 Dielectric objects $\left(\epsilon_{r}=3.5\right)$ are placed on the back (square 196 cross section) and on the right arm. The OUT is displaced 197 from the position $x=-0.8 \mathrm{~m}$ to $x=0.8 \mathrm{~m}$ in $40-\mathrm{cm}$ steps 198 obtaining $N_{\text {pos }}=5$ intermediate positions. For every position, 199 the ten transmitters are sequentially activated and the scattered 200 field is collected in the receiving points. A realistic compo- 201 sition of the human body tissue is considered [16], using a 202 finite-difference frequency-domain (FDFD) code [17], [18] to 203 calculate the scattered field for every transmitter and every OUT 204 position. FDFD simulation results have confirmed that, due to 205 the high conductivity of the skin in the frequency band of inter- 206 est, the assumption that the OUT is a perfect electric conductor 207 (PEC) is a good approximation for most cases. 208

The data are then used to create one reflectivity image for 209 each intermediate position, $\rho^{p}$ according to (1) and (2). The 210 imaging domain is an $(X, Y)=(0.4,0.6) \mathrm{m}$ rectangle, dis- 211 cretized in $81 \times 121$ pixels and centered in $\left(x_{p}^{\prime}, y_{p}^{\prime}, z_{p}^{\prime}\right)$. In this 212 case, the computational cost is low and the image is recovered 213 using the standard backprogation algorithm in (1). For every $p$ th 214 OUT position and $t$ th active transmitter, the image is recovered 215 in about $1 \mathrm{~s}$ using a conventional laptop (2.5-GHZ CPU and 4- 216 GB RAM memory). As the 2-D imaging code is not parallelized 217 yet, it takes about $50 \mathrm{~s}$ for the entire reconstruction. 218

The obtained images for two different active transmitters 219 when the OUT is in each of the intermediate positions are pre- 220 sented in Fig. 3. It is clear that each transmitter allows the 221 reconstruction of different areas of the body depending on its 222 relative position inside the imaging system. The image obtained 223 for the central position, combining the images created using all 224 the transmitters according to (2), is presented in Fig. 4. 

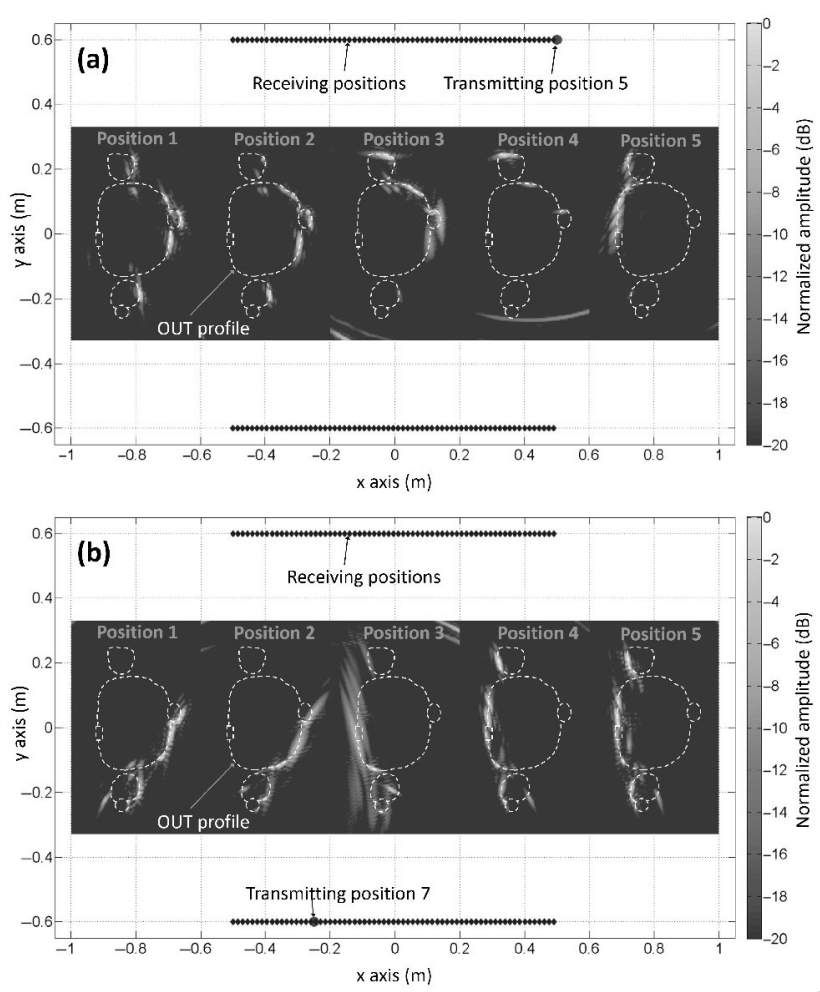

F3:1 Fig. 3. Obtained images (normalized reflectivity amplitude in $\mathrm{dB}$ ) for two difQ1 F3:2 ferent active transmitters and five intermediate positions using the setup in F3:3 Fig. 2. Active transmitters are depicted as red points. Blue points represent F3:4 receivers positions.

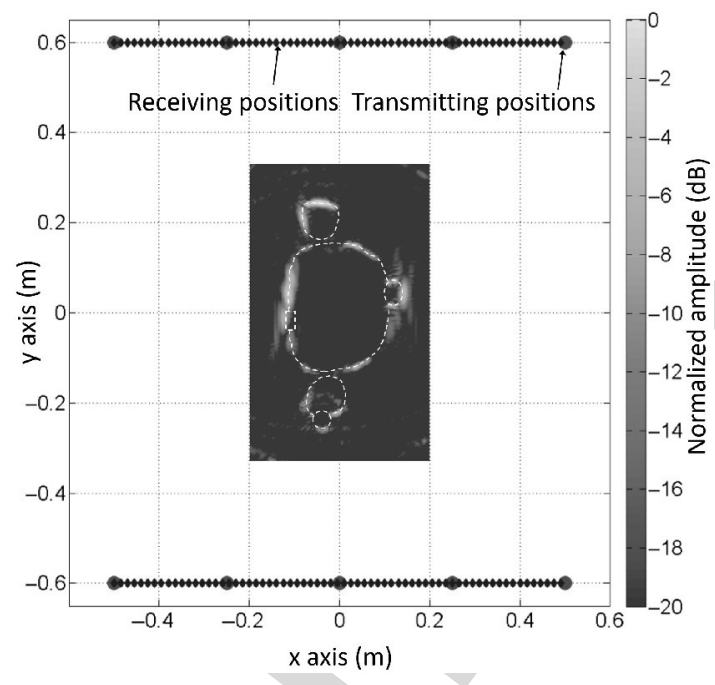

F4:1 Fig. 4. Obtained image when the OUT is in the central position and the image F4:2 is created using all transmitters according to (2).
The reflectivity image created by the system at each position is obtained as

$$
I\left(x^{\prime \prime}, y^{\prime \prime}, z^{\prime \prime}\right)=\sum_{p=1}^{N_{\text {pos }}}\left|\rho\left(x^{\prime}-x_{p}^{\prime}, y^{\prime}-y_{p}^{\prime}, z^{\prime}-z_{p}^{\prime}\right)\right|
$$

where the reflectivity of all the positions is centered at the origin of coordinates before being combined. Absolute value is used since the position of the OUT relative to the imaging system can slightly change from position to position, which prevents the
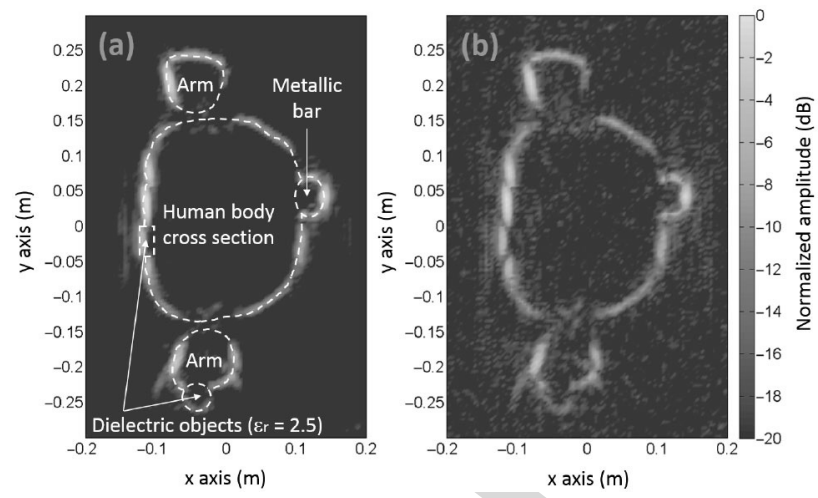

Fig. 5. Recovered OUT profile when combining in amplitude the five images F5:1 (one for each position). (a) $\mathrm{SNR}=10 \mathrm{~dB}$. (b) $\mathrm{SNR}=-20 \mathrm{~dB}$.

combination of the images of each position in amplitude and 232 phase. Fig. 5 presents the final result when the five analyzed 233 positions are combined according to (3), and when the object 234 retains exactly the same configuration for all positions and it is 235 only displaced in the $\mathrm{x}$ direction. This proves the ability of the 236 proposed system to obtain a complete contour reconstruction. 237 In general, the images used for threat detection in a final con- 238 figuration would be the ones generated in each position as the 239 one in Fig. 4.

Combining the information from multiple transmitters and 241 positions also helps to increase the dynamic range of the sys- 242 tem. Sensitivity analysis has been performed: first, the recorded 243 signal strength in the receiving arrays for every transmitting ele- 244 ment and OUT position has been evaluated. The case in which 245 maximum power is recorded corresponds to the OUT at the 246 central position illuminated by the center transmitters. The min- 247 imum power levels are recorded for the OUT in positions 1 or 248 5 illuminated by the closest pair of transmitters, as only a small 249 fraction of the scattered field is collected by the arrays. The 250 received power difference between these two cases is $11 \mathrm{~dB}$. 251

Next, noise has been added to the field samples accord- 252 ing to different signal-to-noise ratio (SNR) levels relative to 253 the maximum-recorded power case. Figs. 3-5(a) correspond to 254 $\mathrm{SNR}=10 \mathrm{~dB}$, and Fig. 5(b) to SNR $=-20 \mathrm{~dB}$. Thanks to the 255 combination of multiple OUT positions and incident directions, 256 the resulting mm-wave imaging system is able to work with low 257 SNR.

The capability of imaging multiple users within the hallway 259 has been also evaluated. For this purpose, the OUT placed at the 260 center position (as in Fig. 4) is considered, but with two more 261 OUTs (with no attached objects) at $x=0.7$ and $x-0.7 \mathrm{~m}$, a 262 scenario that could correspond to a high passenger through- 263 put situation. Due to the use of FDFD simulations, multiple 264 reflections among OUTs are considered. Results are depicted 265 in Fig. 6. It can be noticed that, with respect to Fig. 4, the cen- 266 ter OUT is worse imaged due to the multipath effects. It it also 267 possible to create the image of the front and the back of the 268 OUTs placed at $x=0.7$ and $x=-0.7 \mathrm{~m}$, and these results are 269 not affected by multipath as much as the center OUT.

In order to compare this work with current state of the art 271 systems, Fig. 7 presents the obtained image when the same con- 272 tour is facing a line containing the transmitters and receivers. In 273 


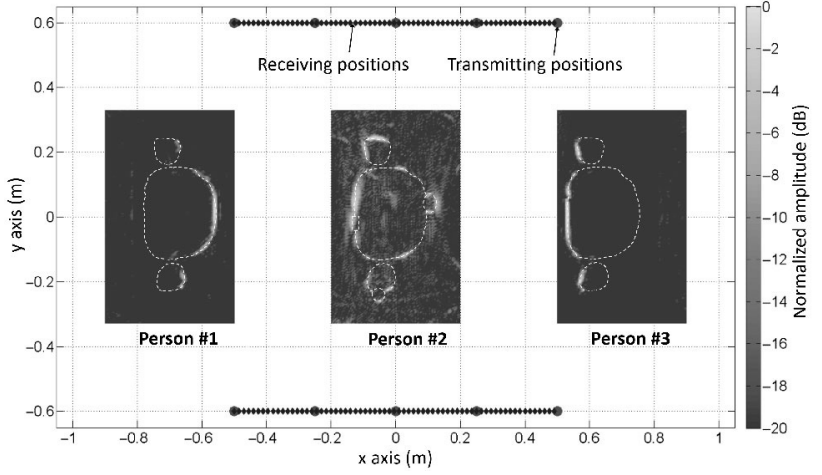

F6:1 Fig. 6. Recovered image for three OUTs placed at the same time in the hallway.

F6:2 The image is created by combining all transmitters according to (2).

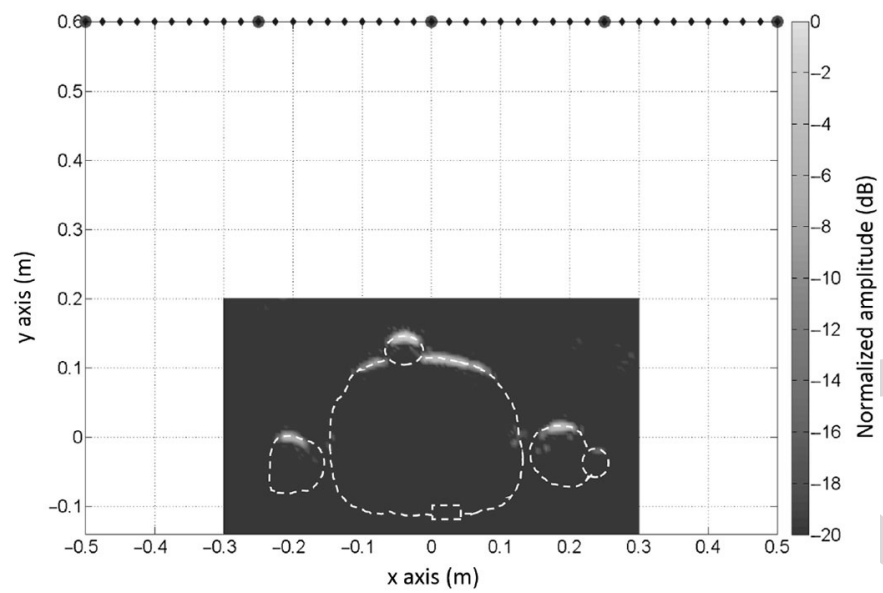

F7:1 Fig. 7. Obtained image using state-of-the-art configurations where transmitters F7:2 and receivers are placed in the same aperture and facing the person under test. F7:3 The image is generated combining the five transmitters according (2). this case, different areas of the front of the contour cannot be recovered and the area that is reconstructed is much smaller than the one of Fig. 4. Concerning detection capabilities, note that the dielectric object placed on the arm is hardly detected in Fig. 7 as the energy is not scattered back to the receiving array. In the case of the on-the-move system, it can be better detected (see Figs. 4 and 5), as it is possible to find a configuration along the conveyor belt in which the energy is reflected in the dielectric-skin transition, then backscattered to one of the receiving arrays.

This 2-D example proves that, in the proposed on-the-move layout, the fact that some of the transmitters and receivers are separated with a subtended angle relative to the person equal or greater than $90^{\circ}$ provides information from all possible wave incident angles.

\section{Validation With Measurements}

The proposed on-the-move imaging concept has been validated with measurements. Ka frequency band $(26.5-40 \mathrm{GHz})$ has been selected to avoid hardware switching between different frequency bands. In order to ensure the maximum illumination within the hallway, WR-28 open-ended waveguides are selected as antennas.

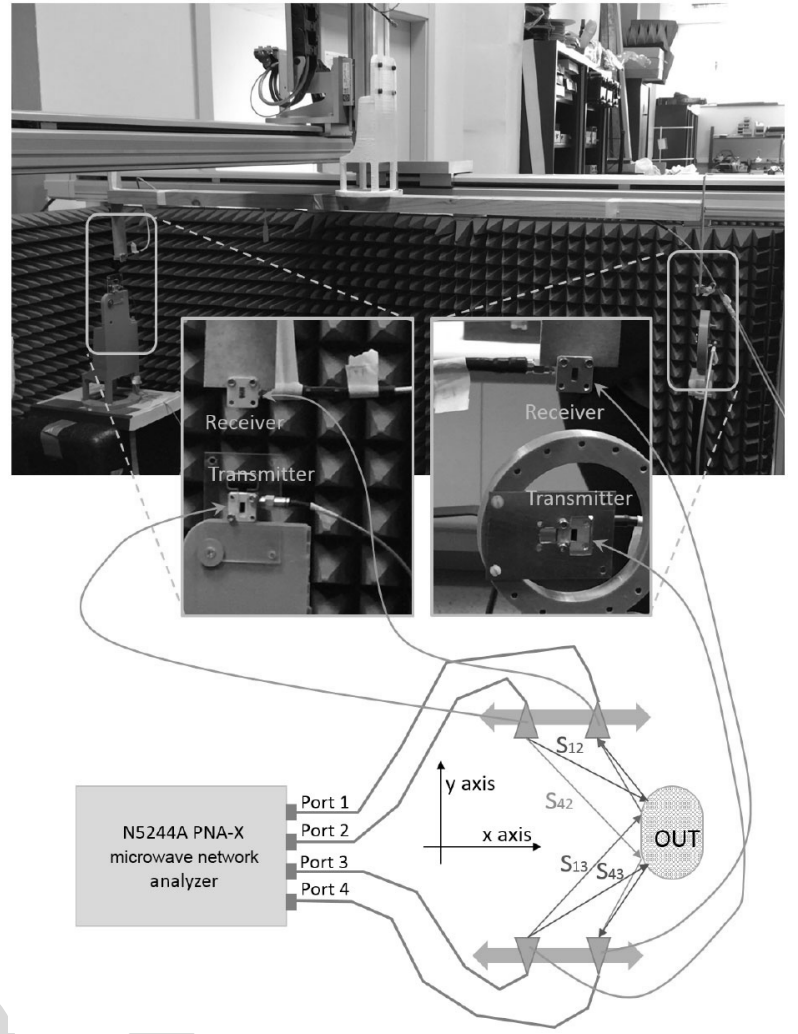

Fig. 8. Ka-band measurement system for on-the-move concept experimental F8:1 validation. WR-28 open-ended waveguides are connected to the vector network F8:2 analyzer ports. Receivers are mounted on a three-axis positioner.

F8:3

The setup is mounted on an XYZ table measurement range 296 [19], so some mechanical restrictions apply to the placement 297 of the OUT, transmitters, and receiving positions (Fig. 8). In 298 order to take advantage of the whole span of the XYZ measure- 299 ment range, scattered field samples are collected in 161 points 300 ranging from $x=-0.6 \mathrm{~m}$ to $x=0.6 \mathrm{~m}$, placed at $Y_{0}=0 \mathrm{~m} 301$ and $Y_{0}=1.3 \mathrm{~m}$. Five transmitting positions are interleaved 302 among the receivers, thus resulting in $N_{t x}=10$ transmitting 303 positions. Transmitters and receivers are separated $5 \mathrm{~cm}$ in 304 height. Horizontal polarization is considered to reduce coupling 305 between transmitter and receiver. The imaging setup is depicted 306 in Fig. 8: two transmitters and two receivers are connected to 307 the ports of a vector network analyzer. The power reference 308 level is $0 \mathrm{dBm}$ for all the ports. For every receiving position 309 along the X-axis, four S-parameters are measured, as shown in 310 Fig. 8, corresponding to the combination of each transmitter 311 with both receivers. $\quad 312$

The positioner of the XYZ table is used to move the receivers 313 from each side of the hallway at the same time, as shown in 314 Fig. 8. The pair of transmitters is manually placed at five posi- 315 tions along the $\mathrm{x}$-axis, using the $\mathrm{XYZ}$ positioner as reference. 316 For every pair of transmitting positions, acquisition time takes 317 $3 \mathrm{~min}$, and therefore, overall acquisition time for every OUT 318 position is $15 \mathrm{~min}$.

The OUT, shown in Fig. 9, is an aluminum foil-covered plas- 320 tic bin with a metallic bar attached to one of the sides. Due 321 to its translation symmetry in z-axis, it allows for 2-D analysis 322 in an XY plane placed at $\left(z=h_{t x}+h_{r x} / 2\right)$, where $h_{t x}$ is the 323 


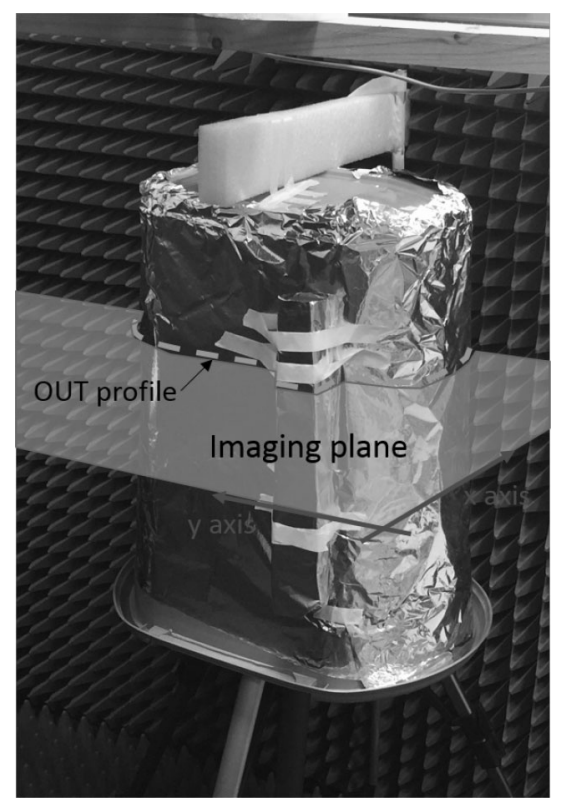

F9:1 Fig. 9. Photograph of the OUT imaged with the proposed experimental setup. F9:2 Receivers are mounted on a three-axis positioner.

height of the transmitters, and $h_{r x}$ the height of the receivers. As mentioned in Section II, using metal to simulate the human body skin in the Ka band is an acceptable approach due to the high conductivity of the skin in mm-wave frequency bands [16]. Three positions of the OUT were considered.

The same data processing as in Section III has been applied. The image obtained for every position, combining the images created using all the transmitters according to (2), is depicted in Fig. 10(a). It can be noticed that, for positions 1 and 3, the front and the back of the OUT are imaged, and the sides of the OUT are visible for position 2 .

Fig. 10(b) presents the final result combining the three OUT positions according to (3), where the OUT profile can be observed. In this case, combination is done taking the displacement of each individual image with respect to the center of the imaging domain. In practical, combination of the radar images for different positions of the person in the hallway can be based on video frames, linking video, and radar images.

In addition to the presented results, the measurement setup has been simulated, aiming to evaluate the correspondence between simulations and measurements. Results for position 2 are compared in Fig. 11. Good agreement between the reconstructed parts of the OUT for simulations and measurements is obtained.

\section{3-D CONFIGURATION}

Next, the extension from 2-D to 3-D is presented. The layout of the proposed on-the-move 3-D system is presented in Fig. 12. The setup is composed of multiple synchronized transmitters and receivers. Lateral receiving apertures of size $(\mathrm{X}, \mathrm{Z})=$ $(1,2) \mathrm{m}$, are placed at $Y_{0}=0.75 \mathrm{~m}$. The size of the panels is chosen to provide an approximated cross-range resolution of $1 \mathrm{~cm}$ along the $\mathrm{z}$-axis and $2 \mathrm{~cm}$ in the $\mathrm{x}$-axis.

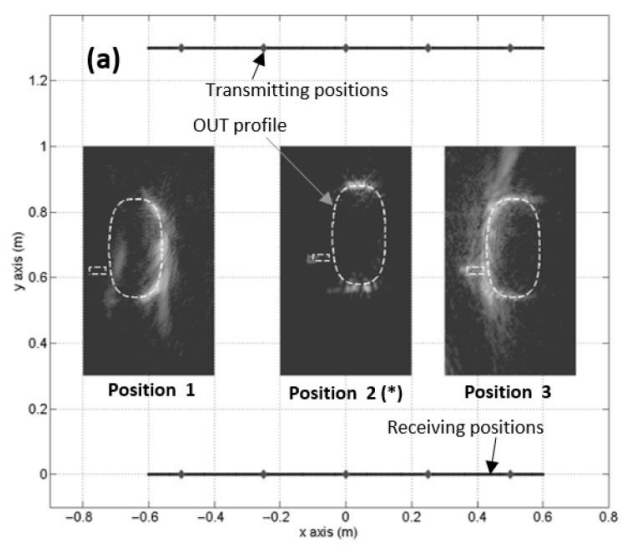

(b)

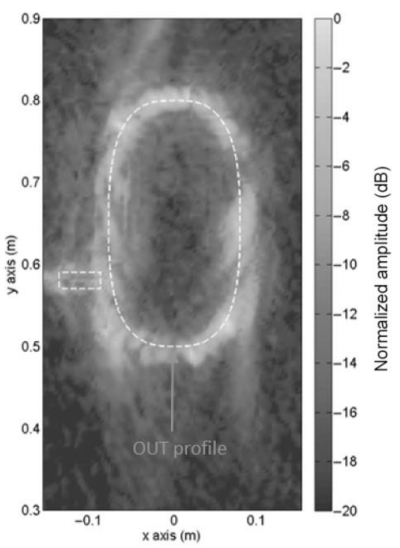

Fig. 10. Recovered OUT profile. (a) Image created on every position using F10:1 all the transmitters according to (2). In the case of position 2, only the cen- F10:2 ter transmitting positions $\left(x_{i n c}^{t}=0 \mathrm{~m}\right.$ ) were available. (b) OUT profile when F10:3 combining in amplitude the three images of (a).

F10:4
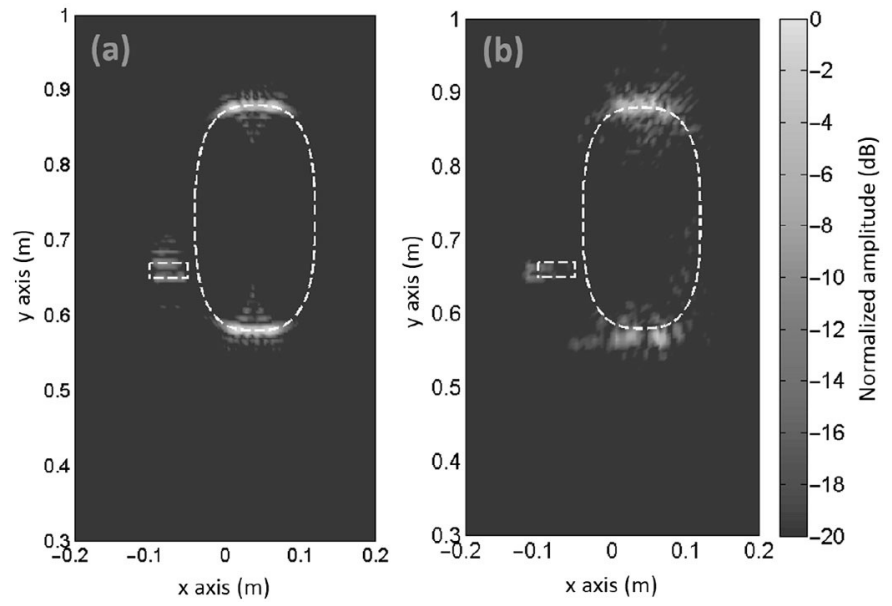

Fig. 11. Recovered OUT profile, position 2 (with center transmitting posi- F11:1 tions). (a) From simulated data. (b) From measurements.

F11:2

For this preliminary setup, Nyquist sampling requirements 356 are considered for the receiving panels, thus acquiring the field 357 in $201 \times 401$ receiving positions per panel. Subsampling tech- 358 niques as presented in [2] and [5] combined with a modified 359 FFT algorithm for multistatic imaging with subsampled arrays 360 can be efficiently applied in this setup to reduce the number 361 of receivers in more than $90 \%$ [2], although this analysis is 362 beyond the scope of this contribution. A $15-\mathrm{GHz}$ bandwidth 363 


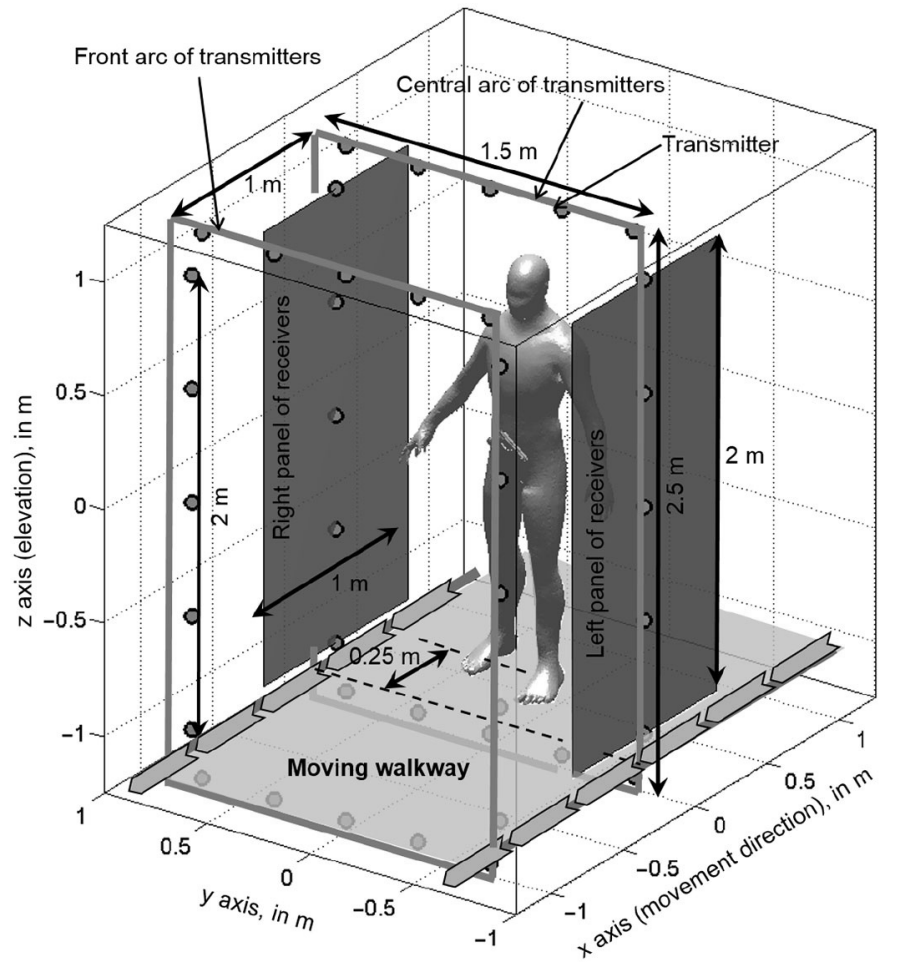

F12:1

F12:2

F12:3

Fig. 12. Layout of the mm-wave scanner for personnel screening. For the sake of simplicity, just two arcs of transmitters, at $\mathrm{x}=0 \mathrm{~m}$, and $\mathrm{x}=-1 \mathrm{~m}$, are considered. The person under test is placed at $\mathrm{x}=0.25 \mathrm{~m}$.

(BW), from 15 to $30 \mathrm{GHz}$, is chosen, similarly to the UWB imaging system described in [5]. This BW provides and approximate range resolution of $1 \mathrm{~cm}$, although, for near-field radar imaging, besides the frequency and aperture size, the final system lateral and range resolutions are given by (2) and (3) of [20], respectively.

Hallway scanner dimensions have been selected to provide a resolution similar to other mm-wave scanners, as shown in Table I. It must be reminded that the number of receiving elements can be reduced in the hallway system.

Concerning processing time, the fastest operational $\mathrm{mm}$ wave imaging systems listed in Table I are capable to provide detection results in less than $5 \mathrm{~s}$, so the scanning process can take up to $10 \mathrm{~s}$ taking into account that the person needs to be placed in a particular position within the scanner. For the presented system, the overall scanning process would be limited by the time the person needs to go through the hallway.

Three arcs of transmitters, centered at $\mathrm{x}=+1,0$, and $-1 \mathrm{~m}$, and each having 20 elements evenly spaced along $y$ - and z-axes, are considered. For the sake of simplicity, only the ones at -1 and $0 \mathrm{~m}$, depicted in Fig. 12, will be considered to obtain the results in this section. Some of the transmitters are placed on top and below the body to ensure the areas with larger curvature (as the top of the chest and shoulders) are reconstructed.

A physical optics (PO) code [21], [22] in combination with a visibility algorithm [23] has been used to predict the parts of the body model in Fig. 12 that are illuminated by every transmitter. Also, PO provides the amount of scattered field collected on the panels. Thus, it is possible to evaluate if a certain layout of transmitters is capable of illuminating the entire person after crossing the hallway and to estimate the field scattered by the (a)

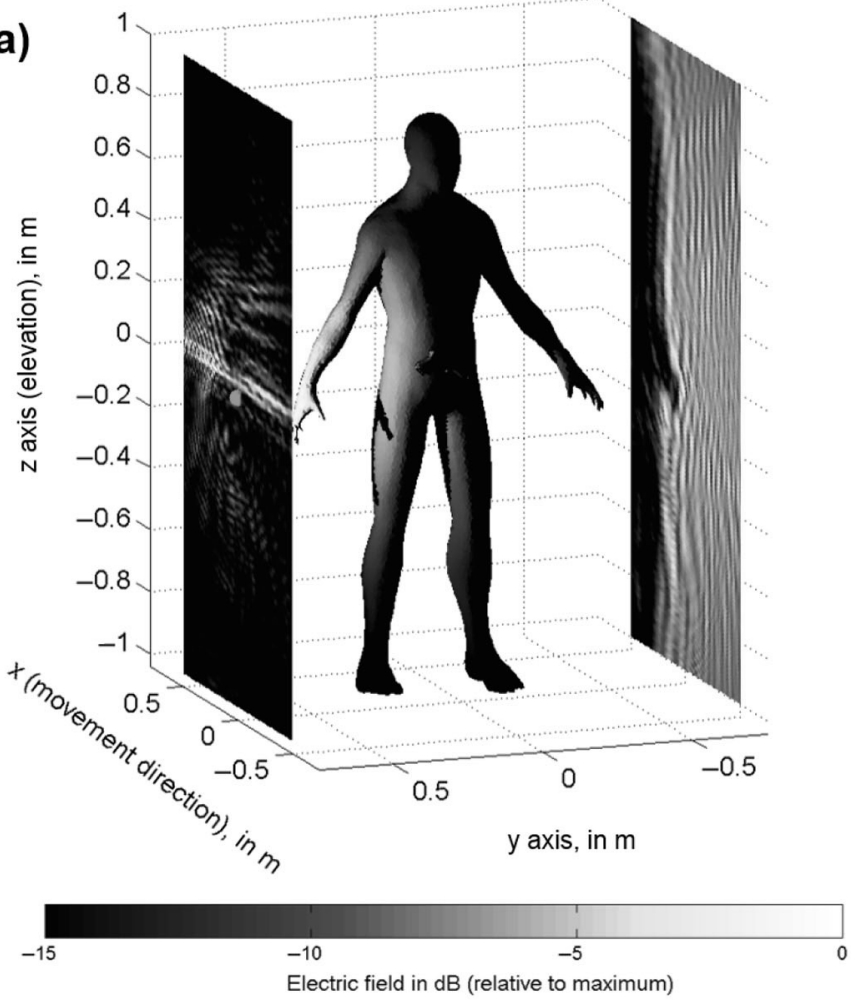

(b)

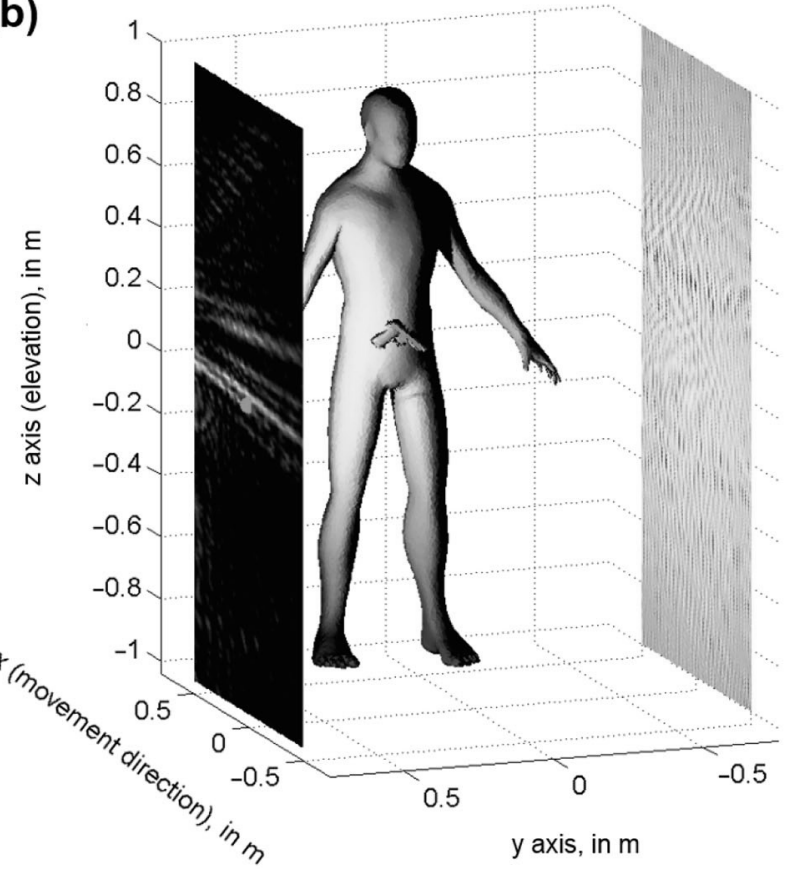

Fig. 13. Examples of human body illumination using one transmitter (high- F13:1 lighted in green) and scattered field on the array panels when the body model is F13:2 centered in (a) $0.25 \mathrm{~m}$ and (b) $0.75 \mathrm{~m}$.

F13:3

illuminated areas on the receiving panels. For these simulations, 395 the human body is assumed to behave as a PEC in the 15-30- 396 $\mathrm{GHz}$ frequency band.

As an example, Figs. 13 and 14 show the regions of the 398 human body under test illuminated by two different transmit- 399 ters, as well as the field received on the lateral panels. Note that, 400 even for a single position of the person in the hallway, different 401 

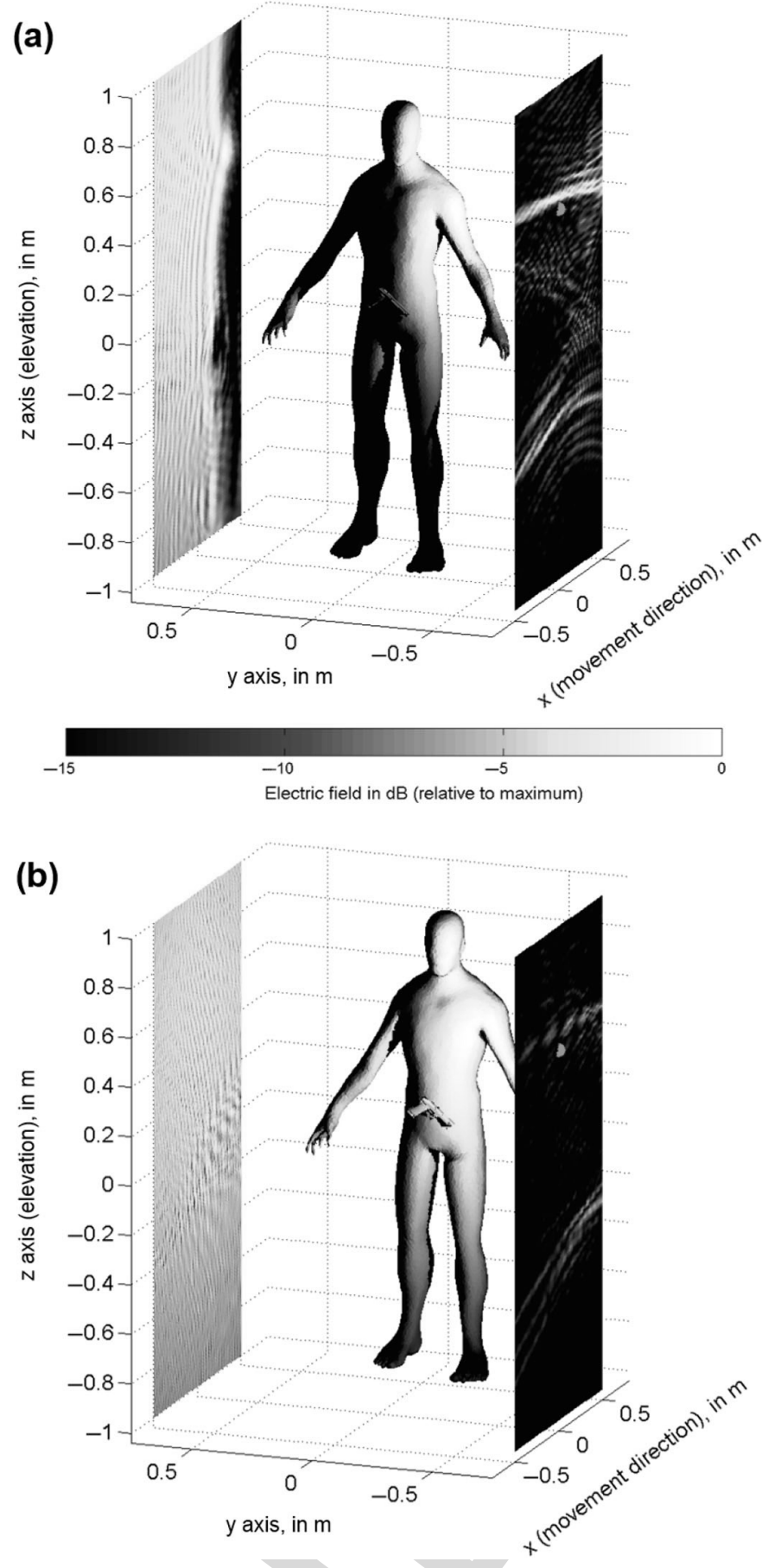

F14:1 Fig. 14. Examples of human body illumination using one transmitter (highF14:2 lighted in green) and scattered field on the array panels when the body model is F14:3 centered in (a) $0.25 \mathrm{~m}$ and (b) $0.75 \mathrm{~m}$. areas of the body are illuminated. This layout increases the amount of information thanks to the spatial diversity of the multistatic illumination.

Regarding the inverse method to create images in this system and due to the large computational cost for the imaging, when the backpropagation is implemented in 3-D, the abovementioned Fourier-based technique for multistatic imaging [9] has been used. The efficient use of fast Fourier transforms (FFT) provides 3-D whole body imaging in almost real time using conventional hardware.

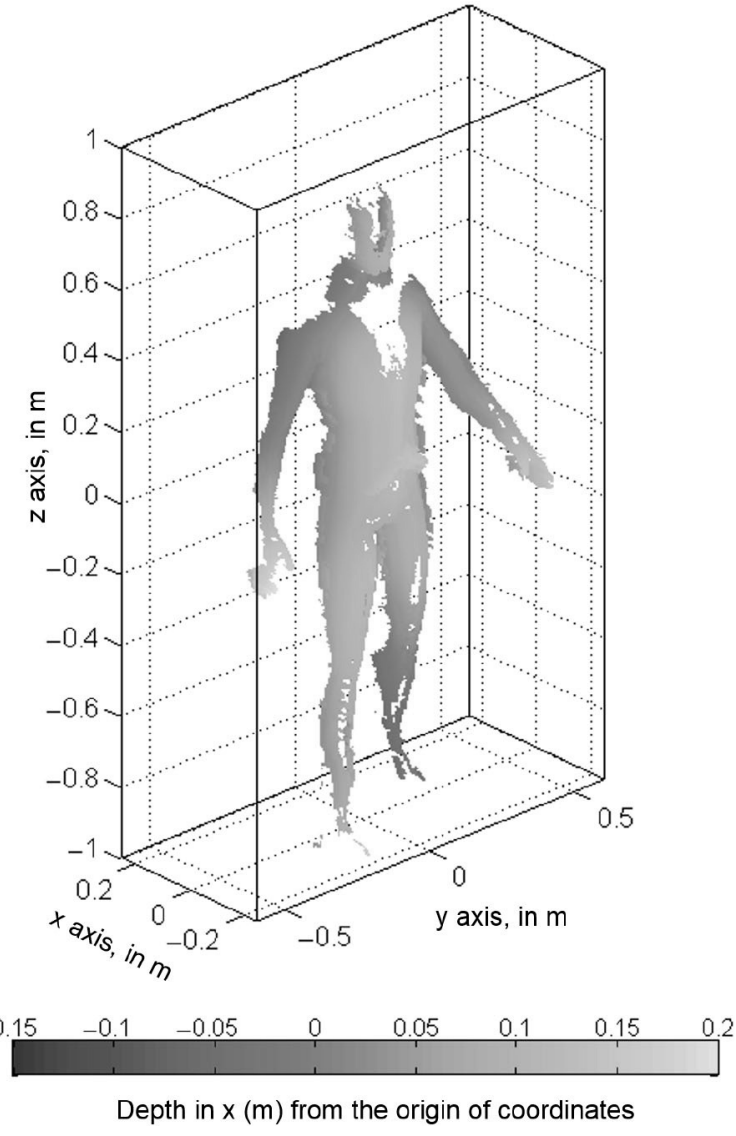

Fig. 15. Person placed at $x=0.25 \mathrm{~m}$. Recovered human body and concealed F15:1 object geometry from backpropagation imaging.

F15:2

As an application example to show the performance of the 412 proposed configuration, an OUT consisting on a person carry- 413 ing a concealed weapon in the belt has been considered. For 414 the sake of simplicity, only two positions are analyzed: person 415 standing at $x=0.25 \mathrm{~m}$ and at $\mathrm{x}=0.75 \mathrm{~m}$. In this example, the 416 goal is to clearly illustrate the different nature of the multistatic 417 information collected on each position, rather than a rigorous 418 reconstruction of the whole body.

For every position, transmitter, and receiving panel, the 420 amount of data to be processed is: $201 \times 401$ spatial samples $\times 421$ 121 frequency samples $\left(=9.75 \times 10^{6}\right.$ scattered field samples $), 422$ which also determines the number of imaging points in the 423 case of Fourier-based imaging [9]. A workstation with 32 cores 424 at $2.1 \mathrm{GHz}$ and 128-GB RAM was used for data processing. 425 Overall calculation time for every transmitter was $30 \mathrm{~s}(1200 \mathrm{~s} \quad 426$ total for the 40 used transmitters). The processing has been 427 done using a sequential Matlab code and has not been optimized 428 for real time imaging yet.

Imaging results are depicted in Figs. 15 and 16, correspond- 430 ing to the person's placement at $\mathrm{x}=0.25 \mathrm{~m}$ and $\mathrm{x}=0.75 \mathrm{~m}, 431$ respectively. Reflectivity points above $-25 \mathrm{~dB}$ with respect to 432 the maximum are coded in depth according to $\mathrm{x}$-axis, allowing 433 the recovery of the human body profile and potential concealed 434 weapons. Comparison of Figs. 15 and 16 provides a clear exam- 435 ple of the on-the-move imaging concept effectiveness. In the 436 case of Fig. 15 (person placed at $x=0.25 \mathrm{~m}$ ), the human body 437 sides and some areas of the chest are imaged by the system. In 438 


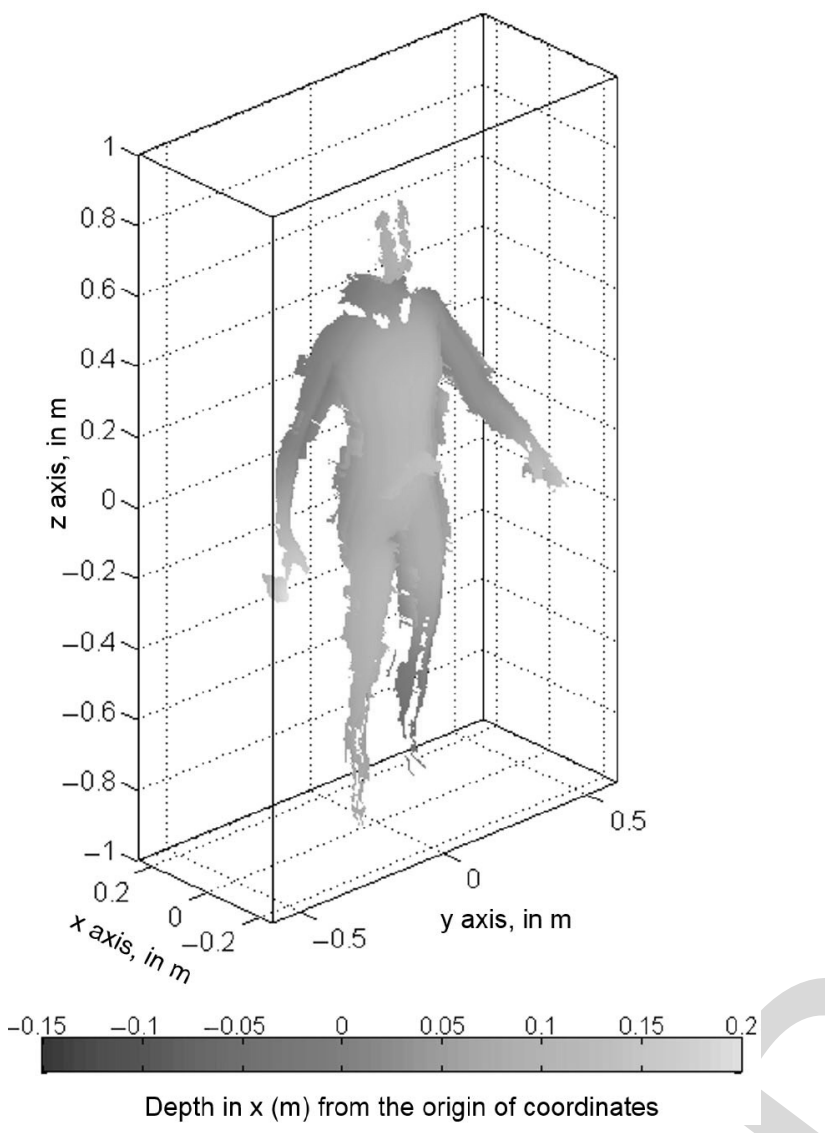

F16:1 Fig. 16. Person placed at $\mathrm{x}=0.75 \mathrm{~m}$. Recovered human body and concealed F16:2 object geometry from backpropagation imaging.

Fig. 16 (person placed at $x=0.75 \mathrm{~m}$ ), the top of the chest and the shoulders are recovered.

In the final system, multiple images, as the two presented examples, can be created and analyzed at video rate to detect any possible threats. Algorithms for mesh generation and automatic thread detection, such as the one used in [8], can be applied.

\section{CONCLUSION}

This work presented a novel concept for personnel scanning in airports and other checkpoints. Unlike the current imaging systems, the proposed system allows for continuous movement of the subject while being scanned; this will greatly increase the system throughput when compared with state-of-the-art systems. This improvement is possible thanks to the use of a fully multistatic radar configuration, where some of the transmitters and receivers are separated with a subtended angle relative to the person greater than 90 degrees to capture information from all possible wave incident angles. In this way, the system is able to create a complete contour reconstruction as the person moves inside the system. The use of a small number of transmitters allows for fast image creation as all the transmitters can be sequentially activated in a short amount of time. 2-D and 3-D simulation-based results confirm the good imaging capabilities of the proposed system; 2-D results have also been validated using measurements. Further work will be related with the setup optimization, including the use of sparse arrays and other tech- 464 niques to reduce the number of receivers, and with experimental 465 validation.

\section{REFERENCES}

[1] IATA. Checkpoint of the Future. Executive Summary [Online]. Available: http://www.iata.org/whatwedo/security/Documents/cof-executivesummary.pdf, accessed on Mar. 16, 2015.

[2] S. S. Ahmed, A. Schiessl, F. Gumbmann, M. Tiebout, S. Methfessel, and L. Schmidt, "Advanced microwave imaging," IEEE Microw. Mag., vol. 13, no. 6, pp. 26-43, Sep./Oct. 2012.

[3] S. S. Ahmed, "Personnel screening with advanced multistatic imaging technology," in Proc. SPIE Defense Secur. Sens., 2013, p. 87150B.

D. Sheen, D. McMakin, and T. Hall, "Three-dimensional millimeter-wave 476 imaging for concealed weapon detection," IEEE Trans. Microw. Theory 477 Techn., vol. 49, no. 9, pp. 1581-1592, Sep. 2001.

468 469 470Q2

471

5] X. Zhuge and A. Yarovoy, "A sparse aperture MIMO-SAR-based UWB 479 imaging system for concealed weapon detection," IEEE Trans. Geosci. 480 Remote Sens., vol. 49, no. 1, pp. 509-518, Jan. 2011.

[6] D. M. Sheen, D. L. McMakin, and T. E. Hall, "Combined illumination cylindrical millimeter-wave imaging technique for concealed weapon detection," in Proc. AeroSense, 2000, pp. 52-60. J. A. Martinez, F. Las-Heras, and C. M. Rappaport, "On the use of 486 compressed sensing techniques for improving multistatic millimeter- 487 wave portal-based personnel screening," IEEE Trans. Antennas Propag., 488 vol. 62, no. 1, pp. 494-499, Jan. 2014.

[8] B. Gonzalez-Valdes, Y. Alvarez-Lopez, J. A Martinez-Lorenzo, F. Las 490 Heras Andres, and C. M. Rappaport, "On the use of improved imag- 491 ing techniques for the development of a multistatic three-dimensional 492 millimeter-wave portal for personnel screening," Prog. Electromagn. 493 Res., vol. 138, pp. 83-98, 2013.

[9] Y. Alvarez et al., "Fourier-based imaging for multistatic radar systems," 495 IEEE Trans. Microw. Theory Techn., vol. 62, no. 8, pp. 1798-1810, Aug. 496 2014.

[10] G. Yates, A. Horne, A. Blake, and R. Middleton, "Bistatic SAR image 498 formation," Inst. Elect. Eng. Proc. Radar Sonar Navigat., vol. 153, no. 3, 499 pp. 208-213, Jun. 2006.

[11] R. Burkholder, I. Gupta, and J. Johnson, "Comparison of monostatic and bistatic radar images," IEEE Trans. Antennas Propag. Mag., vol. 45, no. 3 , pp. 41-50, Jun. 2003

[12] B. Gonzalez-Valdes, C. Rappaport, and J. A. Lorenzo-Martinez, "Onthe-move active millimeter wave interrogation system using a hallway of multiple transmitters and receivers," in Proc. IEEE Antennas Propag. Soc. Int. Symp. (APSURSI), 2014, pp. 1107-1108. move millimiter wave interrogation system with a hallway of multiple transmitters and receivers," U.S. Patent 14562 094, Dec. 5, 2014.

M. Soumekh, "Bistatic synthetic aperture radar inversion with application 511 in dynamic object imaging," IEEE Trans. Signal Process., vol. 39, no. 9, 512 pp. 2044-2055, Sep. 1991.

[15] Y. Alvarez, J. Martinez, F. Las-Heras, and C. Rappaport, "An inverse 514 fast multipole method for imaging applications," IEEE Antennas Wireless 515 Propag. Lett., vol. 10, pp. 1259-1262, Nov. 2011.

[16] D. Andreuccetti, R. Fossi, and C. Petrucci, "An Internet resource for the 517 calculation of the dielectric properties of body tissues in the frequency 518 range $10 \mathrm{~Hz}-100 \mathrm{GHz}$," Internet document, 1997 [Online]. Available: 519 http://niremf.ifac.cnr.it/tissprop/, accessed on Sep. 15, 2015, IFAC-CNR, 520 Florence, Italy, 1997, based on data published by C. Gabriel et al. in 1996. 521

[17] A. W. Morgenthaler and C. M. Rappaport, "Scattering from lossy dielec- 522 tric objects buried beneath randomly rough ground: Validating the semi- 523 analytic mode matching algorithm with 2-D FDFD," IEEE Trans. Geosci. 524 Remote Sens., vol. 39, no. 11, pp. 2421-2428, Nov. 2001.

[18] C. M. Rappaport, Q. Dong, E. Bishop, A. Morgenthaler, and 526 M. E. Kilmer, "Finite difference frequency domain (FDFD) modeling of 527 two dimensional TE wave propagation," in Proc. URSI Symp. Conf., Pisa, 528 Italy, 2004.

[19] A. Arboleya, Y. Alvarez, and F. Las-Heras, "Millimeter and submillimeter planar measurement setup," in Proc. IEEE Antennas Propag. Soc. Int. Symp. (APSURSI), 2013, pp. 1-2.

[20] S. S. Ahmed, A. Schiessl, and L.-P. Schmidt, "A novel active real-time digital-beamforming imager for personnel screening," in Proc. 9th Eur. Conf. Synth. Aperture Radar (EUSAR), Apr. 2012, pp. 178-181. 
[21] J. Meana, J. Martinez-Lorenzo, F. Las-Heras, and C. Rappaport, "Wave scattering by dielectric and lossy materials using the modified equivalent current approximation (MECA)," IEEE Trans. Antennas Propag., vol. 58, no. 11, pp. 3757-3761, Nov. 2010.

[22] L. E. Tirado, J. A. Martinez-Lorenzo, B. Gonzalez-Valdes, C. Rappaport, O. Rubinos-Lopez, and H. Gomez-Sousa, "GPU implementation of the modified equivalent current approximation (MECA) method," Appl. Comput. Electromagn. Soc. J., no. 9, Sep. 2012.

[23] J. Gutiérrez Meana, F. L. Las Heras Andrés, and J. Á. Martínez Lorenzo, "A comparison among fast visibility algorithms applied to computational electromagnetics," Appl. Comput. Electromagn. Soc. J., 2009.

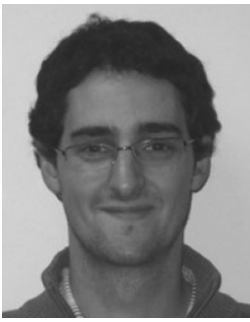

Borja Gonzalez-Valdes (M'xx) received the B.S and $\mathrm{Ph} . \mathrm{D}$. degrees in electrical engineering from the University of Vigo, Vigo, Spain, in 2006 and 2010, respectively.

From 2006 to 2010, he was with the Antenna and Optical Communications Group, University of Vigo. From 2008 to 2009, he was a Visiting Researcher with the Gordon Center for Subsurface Sensing \& Imaging Systems, Northeastern University, Boston, MA, USA. In 2011, he joined the Awareness and Localization of Explosives-Related Threats Center of Excellence, Northeastern University. Since 2015, he has been a Postdoctoral Researcher affiliated with the AtlantTIC Research Center, University of Vigo. His research interests include antenna design, inverse scattering, radar, advanced imaging techniques, and $\mathrm{THz}$ technology.

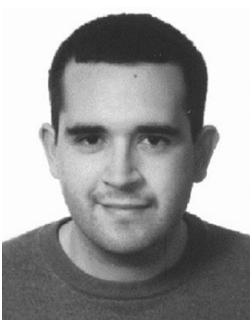

Yuri Álvarez (S'06-M'09-SM'15) was born in Langreo, Spain, in 1983. He received the M.S. and $\mathrm{Ph} . \mathrm{D}$. degrees in telecommunication engineering from the University of Oviedo, Gijn, Spain, in 2006 and 2009, respectively.

He was a Visiting Scholar at the Department of Electrical Engineering and Computer Science, Syracuse University, Syracuse, NY, USA, in 2006 and 2008; a Visiting Postdoc at the Gordon Center for Subsurface Sensing and Imaging Systems (CenSSIS)ALERT (Awareness and Localization of Explosive Related Threats) Center of Excellence, Northeastern University, Boston, MA, USA, from 2011 to 2014; and a Visiting Postdoc at ELEDIA Research Center, Trento, Italy, in 2015. He is currently an Assistant Professor with the Signal Theory and Communications, University of Oviedo, Gijn, Spain. His research interests include antenna diagnostics, antenna measurement techniques, RF techniques for indoor location, inverse scattering and imaging techniques, and phaseless methods for antenna diagnostics and imaging.

Dr. Alvarez was the recipient of the 2011 Regional and National Awards to the Best Ph.D. Thesis on Telecommunication Engineering (category: security and defense).

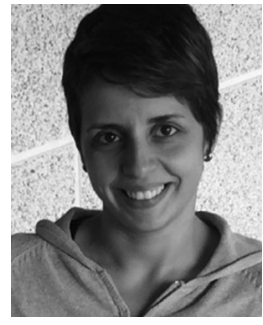

Yolanda Rodriguez-Vaqueiro ( $S^{\prime} \mathrm{xx}$ ) received the B.S. and M.S. degrees in electrical engineering from the University of Vigo, Vigo, Spain, in 2009, and the $\mathrm{Ph} . \mathrm{D}$. degree in electrical engineering from Northeastern University, Boston, MA, USA, in 2015 (after defending her thesis: Compressive Sensing for Electromagnetic Imaging Using a Nesterov-Based Algorithm).

She is a Postdoctoral Researcher affiliated with the AtlantTIC Research Center, University of Vigo. In 2011, she obtained a Research Assistant grant from the ALERT (Awareness and Localization of Explosive Related Threats) Center of Excellence, Northeastern University. She was also granted as a Junior Researcher with the University of Vigo.

Dr. Rodriguez-Vaqueiro was the recipient of the Research-Impact Award by the Department of Electrical and Computer Engineering, Northeastern University (for her work during the Ph.D. studies), the Best Paper Award in the 2012 IEEE Homeland Security Conference, Honorable Mention in the Student Paper Competition in the 2013 IEEE APS/URSI Conference, the Best Paper Award in the 2014 European Conference on Antennas and Propagation, the Burke/Yannas Award to the most original research study in the field of bioengineering in the 2015 American Burn Association (ABA) Meeting, and the Research-Impact Award by the Department of Electrical and Computer Engineering, Northeastern University, in May 2015.

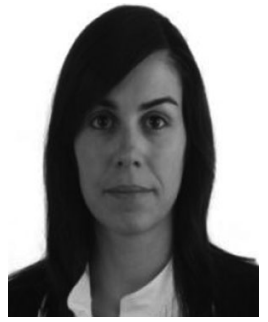

Ana Arboleya-Arboleya received the M.Sc. 607 degree in telecommunication engineering from the 608 University of Oviedo, Oviedo, Spain, in 2009, where 609 she is currently pursuing the Ph.D. degree in telecom- 610 munication engineering. Since 2008, she has been 611 a Research Assistant within the Signal Theory and 612 Communications Research Group, TSC-UNIOVI, 613 Department of Electrical Engineering, University of 614 Oviedo. She was a Visiting Scholar in 2014 and 2015615 at the Department of Radio Science and Engineering 616 and MilliLab, Aalto University, Espoo, Finland. Her 617 research interests include antenna diagnostics and measurement systems and 618 techniques and high-frequency imaging techniques and applications.

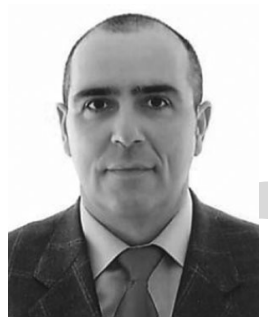

Antonio García-Pino (S'87-M'89-SM'05) was 620 born in Valdemoro, Madrid, Spain, in 1962. He 621 received the M.S. and Ph.D. degrees in telecommuni- 622 cations engineering from the Polytechnic University 623 of Madrid (UPM), Madrid, Spain, in 1985 and 1989, 624 respectively.

From 1985 to 1989, he was a Research Assistant 626 with the Radiation Group, UPM. He joined as 627 an Associate Professor with the Department of 628 Technologies of Communications, University of 629 Vigo, Vigo, Spain, in 1989, becoming Full Professor 630 in 1994. In 1993, he was a Visiting Researcher at the Center for 631 Electromagnetics Research, Northeastem University, Boston, MA, USA. From 632 2006 to 2010, he was the Vice-Rector of Academic Organization and Faculty, 633 and currently, he is the Director of the International Doctoral School, both at 634 University of Vigo. His research interests include shaped-reflector antennas for 635 communication and radar applications, high-frequency backscattering, compu- 636 tational electromagnetics, and THz technology. In these topics, he has authored 637 more than 100 technical papers in journal and conferences and he has been an 638 advisor of $14 \mathrm{Ph} . \mathrm{D}$. thesis.

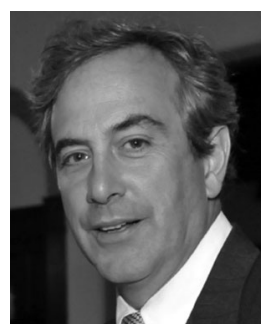

Carey M. Rappaport (SM'96-F'06) received the 640 S.B. degree in mathematics, the S.B., S.M., and E.E. 6 degrees in electrical engineering in 1982, and the $\mathrm{Ph} . \mathrm{D}$. degree in electrical engineering in 1987 from the Massachusetts Institute of Technology (MIT), Cambridge, MA, USA.

He was a Teaching and Research Assistant with MIT from 1981 until 1987, and during the sum- 647 mers at COMSAT Labs, Clarksburg, MD, USA, 648 and the Aerospace Corp., El Segundo, CA, USA. 649 $\mathrm{He}$ joined the faculty at Northeastern University, 650 Boston, MA, USA, in 1987. He has been a Professor of Electrical and 651 Computer Engineering since July 2000. In 2011, he was appointed as a 652 College of Engineering Distinguished Professor. In fall 1995, he was a 653 Visiting Professor of Electrical Engineering at the Electromagnetics Institute, 654 Technical University of Denmark, Lyngby, Denmark, as part of the W. Fulbright 655 International Scholar Program. In the second half of 2005, he was a Visiting 656 Research Scientist at the Commonwealth Scientific Industrial and Research 657 Organisation (CSIRO), Epping, Australia. He has consulted for CACI, Alion 658 Science and Technology, Inc., Geo-Centers, Inc., PPG, Inc., and several munic- 659 ipalities on wave propagation and modeling, and microwave heating and safety. 660 $\mathrm{He}$ was the Principal Investigator of an ARO-sponsored Multidisciplinary 661 University Research Initiative on Humanitarian Demining, the Co-Principal 662 Investigator of the NSF-sponsored Engineering Research Center for Subsurface 663 Sensing and Imaging Systems (CenSSIS), and the Co-Principal Investigator 664 and Deputy Director of the DHS-sponsored Awareness and Localization of 665 Explosive Related Threats (ALERT) Center of Excellence. He has authored 666 more than 400 technical journal and conference papers in the areas of 667 microwave antenna design, electromagnetic wave propagation and scattering 668 computation, and bioelectromagnetics, and has received two reflector antenna 669 patents, two biomedical device patents, and four subsurface sensing device 670 patents.

Prof. Rappaport is a member of Sigma Xi and Eta Kappa Nu professional 672 honorary societies. He was the recipient of the IEEE Antenna and Propagation 673 Society's H. A. Wheeler Award for the Best Applications Paper, as a Student in 674 1986.

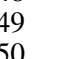

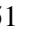

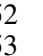
(at) (n)

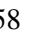
(n)

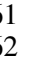
(n) (a) (n) (n) 
676

677

678

679

680

681

682

683

684

685

686

687

688

689

690

691

692

693

694

695

696

697

698

699

700

701

702

703

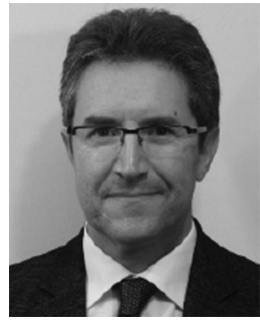

Fernando Las-Heras (M'86-SM'08) received the M.S. and Ph.D. degrees in telecommunication engineering from the Technical University of Madrid (UPM), Madrid, Spain, in 1987 and 1990, respectively.

He was a National Graduate Research Fellow (1988-1990), and he held a position of Associate Professor with the Department of Signal, Systems, and Radiocommunications, UPM (1991-2000). From December 2003, he holds a Full Professor position with the University of Oviedo, Oviedo, Spain, where he was the Vice-Dean for Telecommunication Engineering, Technical School of Engineering, Gijón, Spain (2004-2008). As of 2001, he was the Head of the Research Group Signal Theory and Communications TSC-UNIOVI, Department of Electrical Engineering, University of Oviedo. He was a Visiting Lecturer at the National University of Engineering, Rímac Lima, Peru, in 1996, a Visiting Researcher at Syracuse University, Syracuse, NY, USA, in 2000, and a short-term Visiting Lecturer at ESIGELEC, France, from 2005 to 2011. He held the Telefónica Chair on RF Technologies, ICTs applied to Environment and ICTs and Smartcities with the University of Oviedo (2005-2015). He has authored more than 300 articles published in academic journals and proceedings of international conferences, mainly in the areas of antenna design and the inverse electromagnetic problem with applications in diagnostic, measurement and synthesis of antennas, phaseless techniques, propagation, and microwave to $\mathrm{THz}$ imaging and localization, as well as in engineering education.

Dr. Las-Heras was a Member of the Board of Directors of the IEEE Spain Section (2012-2015), and from 2010, he was a Member of the Science, Technology, and Innovation Council of Asturias, Asturias, Spain.

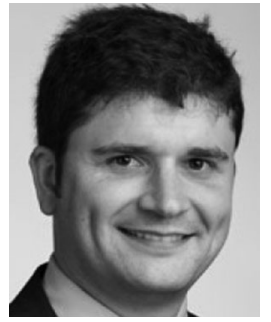

Jose A. Martinez-Lorenzo (M'xx) received the 704 B.S./M.S. degree in 2002 and the Ph.D. degree in 705 2005 from the University of Vigo, Vigo, Spain, both 706 in electrical engineering.

$\mathrm{He}$ joined the faculty at University of Oviedo, 708 Gijon, Spain, in 2004, where he was an Assistant 709 Professor with the Department of Signal Theory 710 and Communications. In 2006, he joined Bernard 711 M. Gordon Center for Subsurface Sensing and 712 Imaging Systems, Northeastern University, Boston, 713 MA, USA. In 2010, he was a Research Assistant 714 Professor with the Department of ECE, Northeastern University. Since August 715 2013, he has been held a joint appointment with the Departments of MIE 716 and ECE as a Tenure-Track Assistant Professor. He is an Active Member of 717 Awareness and Localization of Explosives-Related Threats (ALERT) a DHS 718 Center of Excellence awarded to Northeastern University. He has authored 719 more than 140 technical journal and conference papers. His research inter- 720 ests include the understanding, modeling, and solving complex engineering 721 problems, with an emphasis on mechanical and electromagnetic sensing and 722 imaging methods for security and biomedical applications (i.e., explosive 723 detection, breast cancer detection).

Prof. Martinez-Lorenzo has received funding from multiple agencies, includ- 725 ing: DHS, DARPA, NSF, US Army, and the European Space Agency (ESA). He 726 led the team that won the Best Paper Award in the 2012 IEEE Conference on 727 Technologies for Homeland Security, for the paper on a compressed sensing 728 approach for detection of explosive threats at standoff distances using a passive 729 array of scatterers. 


\section{QUERIES}

Q1: Please provide captions for Fig. 3 subparts.

Q2: Please provide year of publication for Ref. [1].

Q3: Please provide page range for Refs. [18], [22], and [23].

Q4: Please provide volume number for Ref. [22] and [23].

Q5: Please provide the membership history (year) of the authors Borja Gonzalez-Valdes, Yolanda Rodriguez-Vaqueiro,and Jose A. Martinez-Lorenzo.

Q6: Please provide year of completion for the S.B. degree in Mathematics, S.B., S.M. degrees in electrical engineering of author "Carey M. Rappaport." 